Review

\title{
Advances and Prospects of Nanotheranostic Technology for Gastric Cancer
}

\author{
Daxiang Cui ${ }^{1}$, Lijun $\mathrm{Ma}^{2}$, Xiao Zhi ${ }^{1}$, Chunlei Zhang ${ }^{1}$ \\ ${ }^{1}$ Institute of Nano Biomedicine and Engineering, Shanghai Engineering Research Center for Intelligent Diagnosis and Treatment \\ Instrument, Department of Instrument Science and Engineering, School of Electronic Information and Electronical Engineering, \\ National Center for Translational Medicine, Collaborative Innovational Center for System Biology, Shanghai Jiao Tong University, \\ 800 Dongchuan Road, Shanghai 200240, P. R. China. \\ ${ }^{2}$ Department of Oncology, Shanghai Renji Hospital, Shanghai Jiao Tong University School of Medicine, 160 Pujian Road, Shanghai \\ 200127, P.R. China.
}

Corresponding author. E-mail: dxcui@sjtu.edu.cn

Received: Oct. 11, 2016; Accepted: Nov. 4, 2016; Published: Nov. 17, 2016.

Citation: Daxiang Cui, Lijun Ma, Xiao Zhi and Chunlei Zhang, Advances and Prospects of Nanotheranostic Technology for Gastric Cancer. Nano Biomed. Eng., 2016, 8(4): 219-239.

DOI: 10.5101/nbe.v8i4.p219-239.
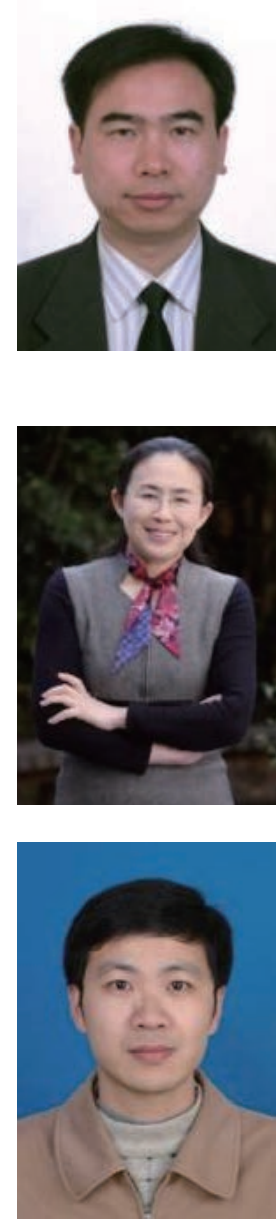

Prof. \& Dr. Daxiang Cui obtained his MD and PhD at the Fourth Military Medical University in 1998, was a post-doctoral fellow in Max Planck Institute for Metals Research from 2001 to 2004, and was a visiting professor in Waseda University from 2007 to 2008. So far he is Distinguished Professor, Changjiang Scholar at Shanghai Jiao Tong University. He has published over 280 papers in international peer-reviewed journals, highindex is 45 . His research interests include controlled synthesis and biosafety evaluation of nanomaterials, nanoparticles-labeling and nano-effects-based tumor theranostic technologies, high efficient drug delivery system and RNA nano-drug.

Prof. \& Dr. Lijun Ma obtained her MD and PhD at the Second Military Medical University in 2004. So far she is Professor and Director at Department of Oncology in Shanghai Tongren Hospital, Shanghai Jiao Tong University Medicine School. Her research interests focus on basis and clinical studies of digestive system tumor theranostics.

Xiao Zhi obtained his PhD at Shanghai Jiao Tong University in 2014, and is a postdoctoral fellow at Shanghai Jiao Tong University from 2014 to 2016. His research interests include nanoparticles-labeling detection technologies, biosensor, microfludic chip and nano-immunologic adjuvant. 


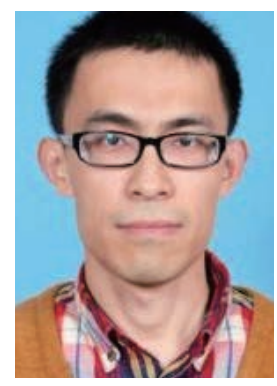

Chunlei Zhang received his BS from Shandong Normal University in 2009, obtained $\mathrm{PhD}$ degree from Institute of Nano Biomedicine and Engineering, Shanghai Jiao Tong University under the supervision of Prof. Daxiang Cui in 2015. Now he worked as a postdoctoral fellow in Shanghai Jiao Tong University. His current research interest is the synthesis of gold and silver nanoclusters, their interactions with biological systems and the applications of gold nanoclusters in biomedical imaging and drug delivery.

\begin{abstract}
Over the past few years, gastric cancer prewarning and early theranostic system have achieved great advances. Gastric cancer theranostic strategies still focus on prediction and prewarning of early gastric cancer, early diagnosis and molecular imaging directed operation therapy, enhanced immunotherapy and killing gastric cancer stem cells to overcome multidrug resistance (MDR). Here we review the main advances in this field over the past few years, explore the clinical translational prospects, and discuss the concepts, issues, approaches, and challenges, with the aim of stimulating a broader interest in developing gastric cancer prewarning and early theranostic system and promoting clinical translation.
\end{abstract}

Keywords: Gastric cancer; Biomarker; Sensor; Nanoprobe; Targeted imaging; Targeted therapy

\section{Introduction}

Gastric cancer (GC) is the fourth most common cancer and the second leading cause of cancerrelated mortality worldwide [1]. Gastric cancer is the second most common cancer, and the third leading cause of cancer-related death in China [2]. Although gastroscopy has been broadly used for screening of early gastric cancer patients, gastric cancer remains very difficult to cure effectively, primarily because most patients present advanced stages of the diseases. Therefore, how to find early gastric cancer has become a great challenge.

In 1999, we first proposed the project of developing gastric cancer prewarning and early diagnosis system [3]. Since 1999, we have been trying to realize gastric cancer prewarning and early diagnosis by screening early gastric cancer biomarkers, developing new detection method of gastric cancer biomarkers, establishing gastric cancer pre-warning database including molecular imaging database, biomarker database and information platform [4, 5]. Up to date, we have achieved great advances.

In recent years, molecular imaging has been and is being actively explored for its clinical applications such as tumor genotyping in vivo, molecular imagingguided targeted theranostics and surgery navigation [6-8]. These advances highly depend on advances of multifunctional nanoprobes, also highly depend on advances of nanomaterials and nanotechnology.

Nanotechnology makes an important contribution towards cancer prevention, diagnosis, imaging and therapy [9]. It not only provides unprecedented capability for carrying multiple diagnostic and therapeutic payloads in the same package, but also facilitates targeting delivery into specific sites across complex biological barriers [10]. The multifunctional integrated system combines different properties such as tumor targeting, imaging and selective therapy in an all-in-one system, which provides more useful multimodal approaches in the battle to fight against cancer.

By far, gastric cancer therapeutic methods mainly include surgery, radiation and chemotherapies, which are generally very effective for early and in situ gastric cancers, but advanced and metastatic cases do not respond to chemo- or radiation therapies [11]. Resistance to chemotherapy-induced apoptosis is a major cause for the failure of conventional therapies. The current prognosis of gastric cancer is very poor with 5-year survivals of less than 24\% [12]. Therefore, how to recognize, track or kill early gastric cancer cells is a great challenge for patients with early gastric cancer.

Current studies show that gastric cancer is not particularly sensitive to traditional therapies, especially 
to chemotherapy agents, which is closely related with gastric cancer stem cells (GCSCs) [13]. Cancer stem cells are a sub-population of cells capable of selfrenewal and unlimited replication to initiate tumors, which have been well characterized in multiple malignancies. CSCs give rise to GC and may be responsible for invasion, metastasis, and resistance to conventional treatment has profound implications for anti-cancer targeted therapies [14]. Therefore, GCSCbased targeted therapy is destined to be one of the most effective anticancer strategies.

Immunological studies highly suggest that the reasons for metastasis and recurrence of GC can be summarized as follows [15]: (I) The intrinsic antigenicity weakness of tumor cells, immunological surveillance of the host cannot identify and eliminate the malignant cells that are distributed out of the resection field and the peripheral lymphoid organs. (II) Immunological surveillance defect or dysfunction of the host. After the traditional therapy was done, the residual tumor cells escaped from immunosurveillance revived again. (III) The substantial toxicities of most traditional curative intents, which is often compromised by the extreme feeble immunity of the patient. Therefore, it is very necessary to develop new therapeutic strategies that could enhance the host immunosurveilliance and/or improve immunogenicity of the tumor cell. Immunological cell therapy should be an alternative choice.

Here we review the main advances of prewarning and early theranostic system of gastric cancer over the past several years, explore the clinical translational prospects, and discuss the concepts, issues, approaches, and challenges, with the aim of improving the clinical translation of prewarning and nano-theranostic technologies of gastric cancer in near future.

\section{Advance of Gastric Cancer Prewarning and Early Theranostic Technologies}

Since President Obama proposed precision medicine project in January 2015, how to realize precision medicine has become a hotspot. Genomics, big data and molecular imaging have become key tools to realize precision medicine. How to realize precise theranostics of gastric cancer has become our concerns. Since we proposed for the first time the concept of gastric cancer prewarning and early diagnosis system in 1999, we have tried to establish gastric cancer prewarning and early diagnosis system by screening early gastric cancer biomarkers, developing new detection method of gastric cancer biomarkers, establishing gastric cancer pre-warning database, including molecular imaging database, biomarker database and information operation platform. Up to date, we have achieved great advances.

\section{Pre-warning gastric cancer patients by SNPs arrays}

With the help of $\mathrm{Fe}_{3} \mathrm{O}_{4}$ magnetic nanoparticles as a solid carrier and an excellent tool for separation, six SNP loci (rs2279115 of BCL2 gene, rs804270 of NEIL2 gene, rs909253 of LTA gene, rs2294008 of PSCA gene, rs3765524 and rs10509670 of PLCE1 gene) were selected to evaluate their relation to gastric cancer risk. Using two kinds of functionalized magnetic nanoparticles and universal tagged arrays, the whole operation procedure including genome DNA extraction and SNP genotyping was performed. All genotypes and allele frequencies were calculated in the cases and controls respectively to analyze their association with gastric cancer risk. Totally 200 pathological samples and 134 normal control subjects were collected. The results demonstrated that four SNP loci (rs2279115, rs804270, rs909253 and rs3765524) showed a potential association with gastric cancer risk, and the other two (rs2294008, rs10509670) possessed no difference/association among cases and controls [16]. The four SNP sites own potential in applications such as gastric cancer risk predication.

\section{Screening early gastric cancer patients by breath analysis based on SERS sensor}

Fourteen volatile organic compound (VOC) biomarkers in breath were identified to distinguish patient with early-stage gastric cancer (EGC) and advanced-stage gastric cancer (AGC) from healthy persons by gas chromatography-mass spectrometry (GC-MS) coupled with solid phase microextraction (SPME). Then a Surface Enhanced Raman Scattering (SERS) sensor for breath analysis was developed to detect those biomarkers as shown in Scheme 1. The SERS sensor is fabricated by in situ synthesis of Au nanoparticles (AuNPs) on reduced graphene oxide (RGO). In the prepared SERS sensor, RGO can selectively adsorb and enrich the identified biomarkers from breath, and AuNPs dispersed on RGO endow this SERS sensor with an effective detection of adsorbed 
biomarkers. Fourteen Raman bands associated with the fourteen biomarkers are selected as the fingerprints of biomarker patterns to distinguish the persons in different states. 200 specimens of real breath samples were analyzed with a sensitivity higher than $83 \%$ and specificity of 92\% [17]. The VOC biomarkers and breath analysis sensor not only can diagnose gastric cancer, but also can distinguish EGC and AGC. This work has a great potential for the clinical translation in diagnosis and stage-determination of gastric cancer in near future.

\section{Simultaneous detection of sera biomarkers by electrochemical microfluidic chip}

How to find early gastric cancer patients is very important to improve the survival rate of patients with gastric cancer. In order to meet the clinical demands for early diagnosis of gastric cancer, we developed a disposable easy-to-use electrochemical microfluidic chip combined with multiple antibodies against six kinds of biomarkers (carcinoembryonic antigen (CEA), carbohydrate antigen 19-9 (CA19-9), Helicobacter pylori CagA protein (H.P.), P53oncoprotein (P53), pepsinogen I (PG I), and PG-II) (Fig. 1). The six kinds of biomarkers related to gastric cancer can be detected sensitively and synchronously in a short time. The specially designed three-electrode system enables cross-contamination to be avoided effectively. The linear ranges of detection of the electrochemical microfluidic chip were as follows: 0.37-90 ng $\mathrm{mL}^{-1}$ for CEA, 10.75-172 $\mathrm{U} \mathrm{mL}^{-1}$ for CA19-9, 10-160 U $\mathrm{L}^{-1}$ for H.P., 35-560 ng $\mathrm{mL}^{-1}$ for P53, 37.5-600 ng $\mathrm{mL}^{-1}$ for $\mathrm{PG}$ I, and 2.5-80 ng $\mathrm{mL}^{-1}$ for PG II. This chip owns better sensitivity compared with enzyme- linked immunosorbent assay (ELISA) results of 394 specimens of gastric cancer sera. Furthermore, we established a multi-index prediction model based on the six kinds of biomarkers for predicting risk of gastric cancer [18]. The developed electrochemical microfluidic chip for detection of multiple biomarkers can be used for early screening of gastric cancer patients, therapeutic evaluation, and real-time dynamic monitoring the progress of gastric cancer in near future.

\section{Gastric cancer CTC capturing and identifying by GMI-based biosensing system}

Circulating tumor cells (CTCs) are those cells that have shed into the vasculature or lymphatics from a primary tumor site and are carried around the body in the circulation. CTCs become seeds for the tumor metastases in vital distant organs, and are responsible for the vast majority of cancer-related deaths. Therefore, CTC counts have become a key technology to diagnose tumor metastasis and progression. A micro-patterned giant magnetoimpedance (GMI) sensor was developed to capture and count gastric cancer CTC cells. RGD-4C peptide coupled chitosan covered superparamagnetic iron oxide particles (RGD$\mathrm{Fe}_{3} \mathrm{O}_{4} @$ chitosan) were used to capture gastric cancer CTC cells [19]. Results demonstrate that this system can efficiently identify targeted gastric cells. Then, a specially designed NiFe-based magnetic biosensing cell chip combined with functionalized magnetic nanoparticles was used for a quick one-step detection of cell surface marker. Magnetic nanoparticles with 20$30 \mathrm{~nm}$ in diameter were prepared by coprecipitation and modified with RGD-4C, and the resultant RGD-

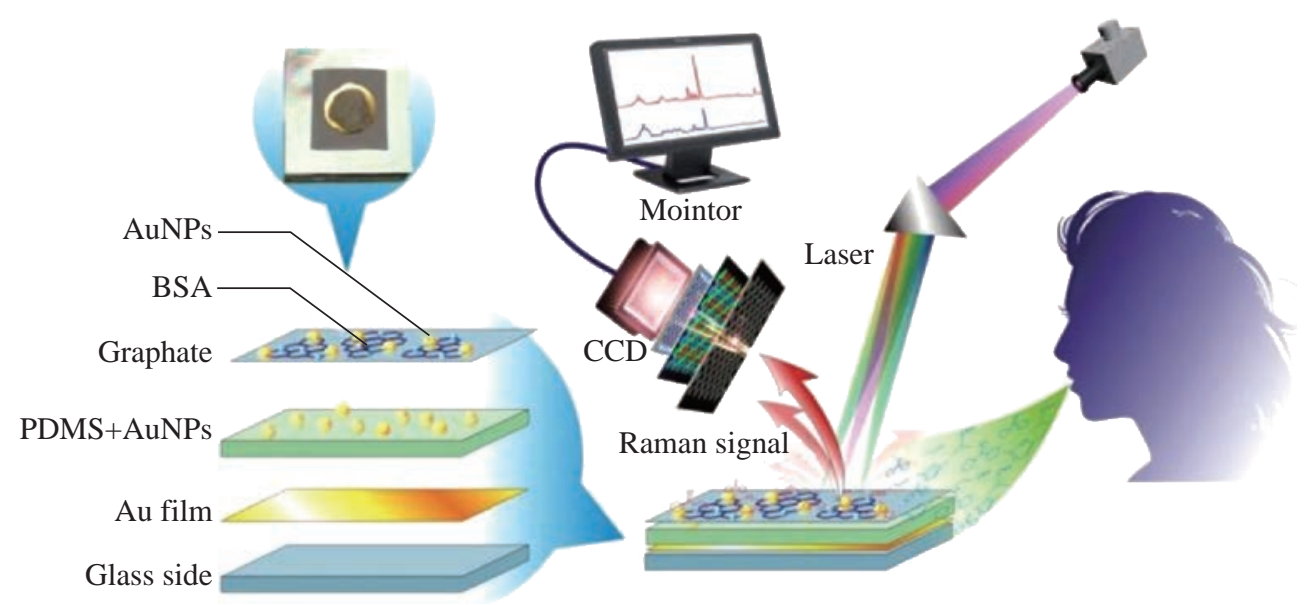

Fig. 1 SERS sensor based on breath-analysis for screening early gastric cancer patients. Reprinted with permission from ACS Press [17]. 

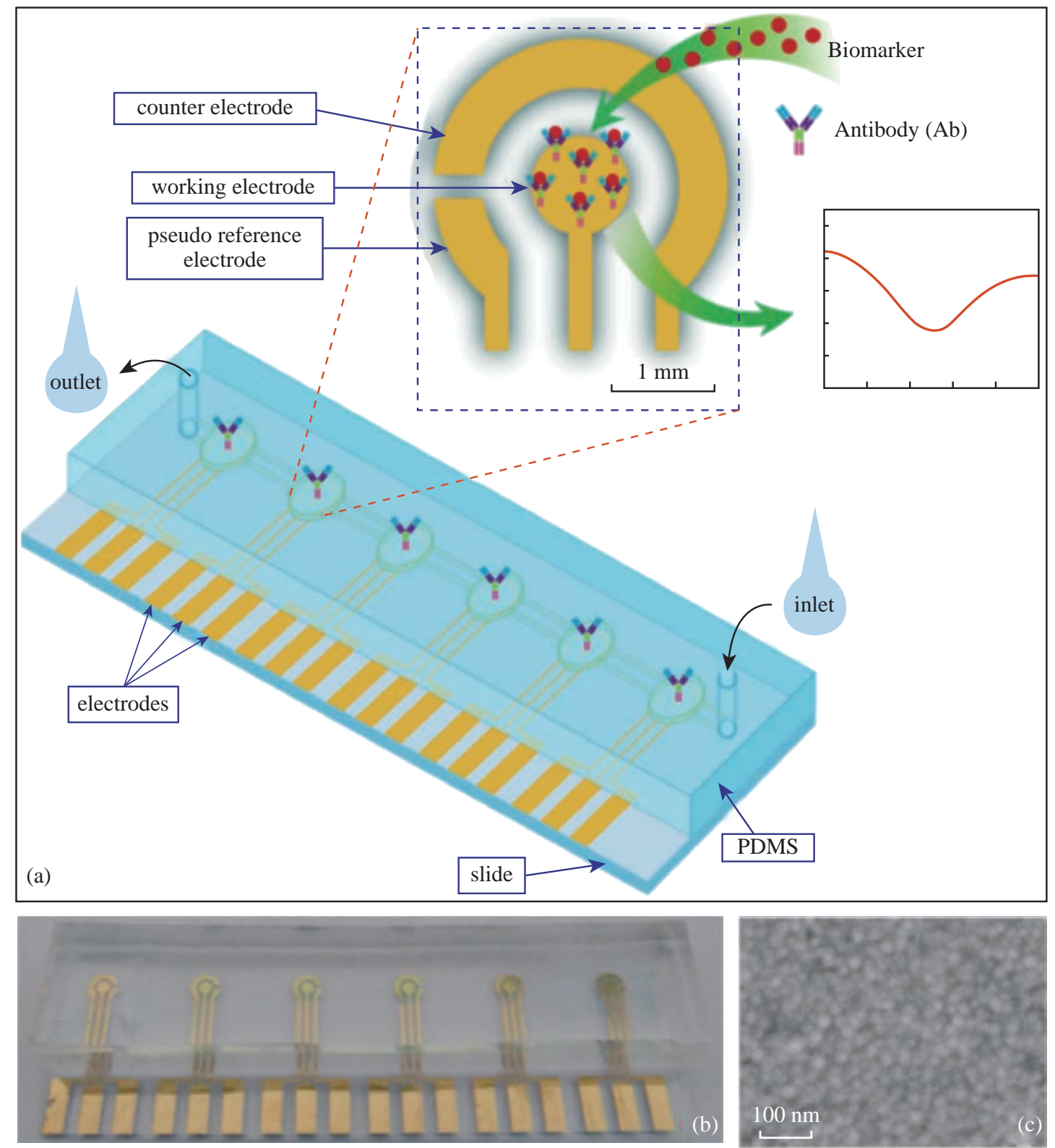

Fig. 2 (a) Schematic illustration of the electrochemical microfluidic chip; (b) Picture of electrochemical microfluidic chip; (c) SEM image of surface of work electrode. Reprinted with permission from Springer press [18].

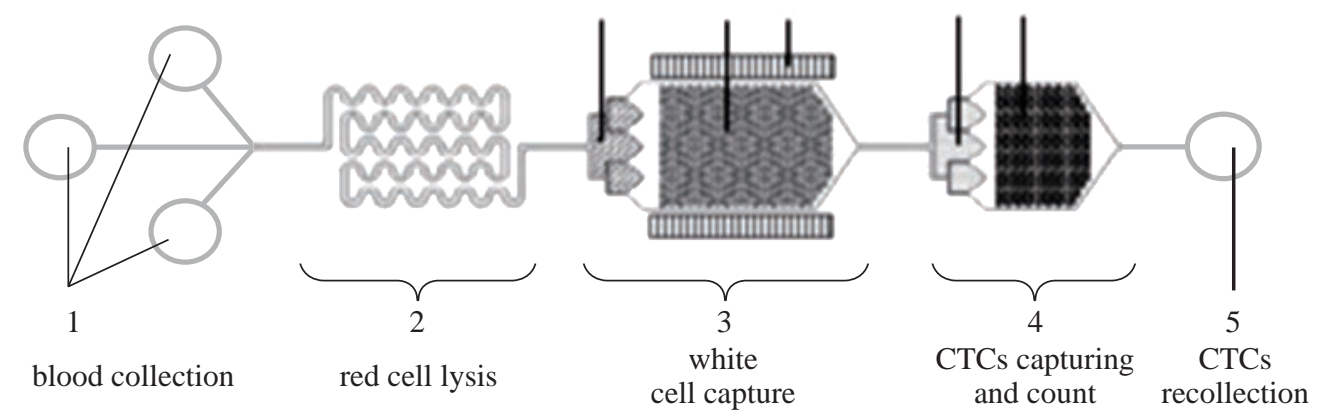

Fig. 3 Schematic illustration of CTC isolation and recollection system.

functionalized magnetic nanoparticles were used for targeting cancer cells cultured on the NiFe-based magnetic biosensing chip and distinguish the amount of cell surface receptor-integrin. This approach has advantages in the qualitative screening test. Compared with traditional method, it is fast, sensitive, low cost, easy-operative, and needs very little human intervention, and can be used for fast clinical cell 
(a) Active targeting of umor blood vessels

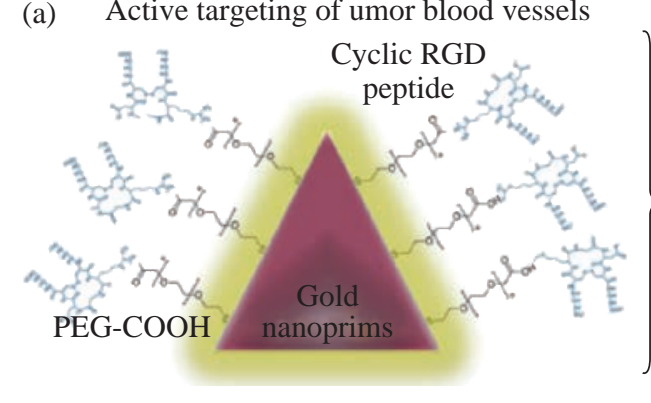

(b)

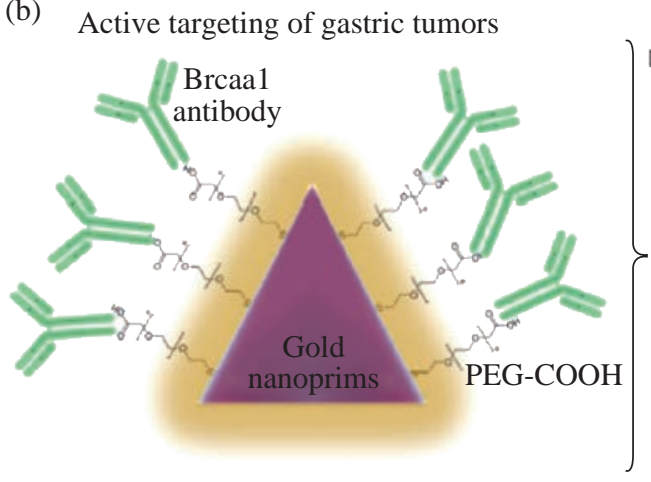

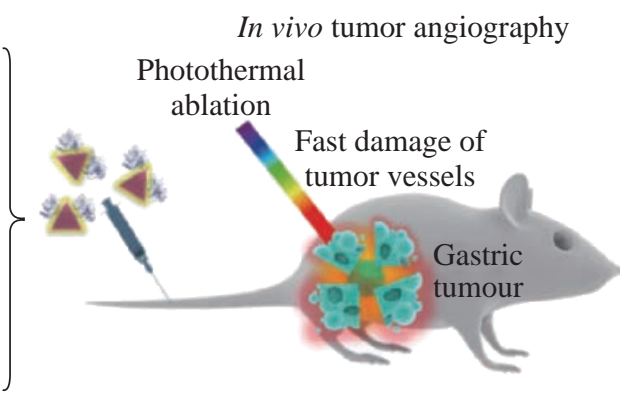

In situ photoacoustic imaging

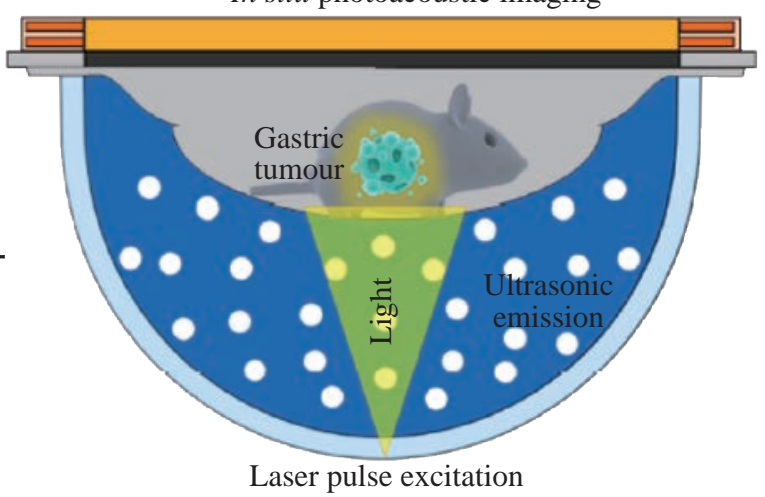

Fig. 4 AuNprs as a hybrid in vivo cancer theranostic platform for in situ photoacoustic imaging, angiography, and localized hyperthermia. (a) Schmatic illustration of the AuNprs functionalized with PEG and a cyclic RGD peptide and their application in photoacousitc angiography and in targeted phototherapy in the tumor vessels; (b) Schematic illustration of the antibody Brcaa1AuNprs and their application in in situ photoacoustic imaging of gastric tumors. Reprinted with permission from Springer Press [22].

surface marker detection, and diagnosis of early cancer [20]. We further developed a device for CTCs capture, counts and recollection. Clinical specimen test showed this system can be used effectively for CTCs isolation and recollection.

\section{Human iPS cells for targeted photoacoustic imaging and phothermal therapy}

How to realize effective accumulation and intratumoral distribution of nanodrug or nanoprobes has become a great challenge for tumor theranostics. AuNRs@SiO $@$ @CXCR4 nanoparticles were prepared, and then the prepared nanoparticles were loaded into the human induced pluripotent stem cells (iPS cells). Due to the prominent optical properties of AuNRs@ $\mathrm{SiO}_{2} @ \mathrm{CXCR} 4$ and remarkable tumor target migration ability of iPS cells, the AuNRs delivery mediated by iPS cells via the AuNRs-iPS was found to have a prolonged retention time and spatially even distribution in MGC803 tumor-bearing nude mice, which was confirmed by photoacoustic tomography and twophoton luminescence. Results showed that the AuNRsiPS system displayed a robust migration capacity to target the tumor site and to improve photothermal therapeutic efficacy on inhibiting the growth of gastric cancer in xenograft mice under a low laser power density [21]. The combination of gold nanorods with human iPS cells as a new theranostic platform paves a novel road for gastric cancer theranostics and holds great promise for clinical translation in the near future.

\section{Gold nanoprisms for photoacoustic imaging, angiography and hyperthermia}

The development of high-resolution nanoscale photoacoustic contrast agents for gastric cancer is an exciting yet challenging technological advance. Antibody (breast cancer-associated antigen 1 (Brcaa1) monoclonal antibody) - and peptide (RGD)functionalized gold nanoprisms (AuNprs) were used as a combinatorial methodology for in situ photoacoustic imaging, angiography, and localized hyperthermia using orthotopic and subcutaneous murine gastric carcinoma models. RGD-conjugated PEGylated AuNprs are available for tumor angiography, and Brcaa1 monoclonal antibody-conjugated PEGylated AuNprs are used for targeting and for in situ imaging of gastric carcinoma in orthotopic tumor models. In situ photoacoustic imaging allowed for anatomical and functional imaging at the tumor site. In vivo tumor angiography imaging showed enhancement of 
the photoacoustic signal in a time-dependent manner. Furthermore, photoacoustic imaging demonstrated that tumor vessels were clearly damaged after localized hyperthermia. These smart antibody/peptide AuNprs can be used as an efficient nanotheranostic platform for in vivo tumor detection with high sensitivity, as well as for tumor targeting therapy [22].

\section{Nanoprobes for genotyping and targeted therapy of in situ gastric cancer}

To realize in situ genotyping of gastric cancer has great clinical requirement. Kras gene mutation and HER2 positive expression are two most common genotypes of gastric cancer tissues. An in vivo universal tool based on AuNPs functionalized with a dye labeled hairpin-DNA, i.e. gold-nanobeacon was developed to inhibit simultaneously gastric cancer cells and metastasis in a murine gastric tumor model following systemic administration via the antisense silencing of a mutant transcript of Kras gene (Fig. 5). The NPs consist of a AuNP core decorated with and a thiolDNA-hairpin labelled with a Cy3 dye. A polyethylene glycol (PEG) is also used as a spacer between the antisense DNA hairpins, increasing the nanostructure stability. Under hairpin configuration, the proximity of the Cy3 dye to the AuNP (that works as quencher) leads to fluorescence quenching. Hybridization of the DNA hairpin to a complementary target (i.e. Kras (a)

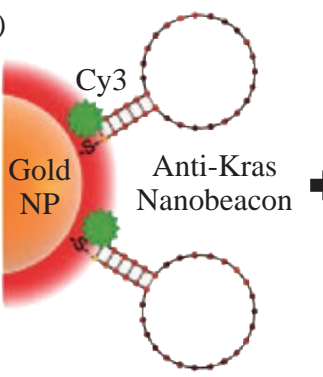

(b)

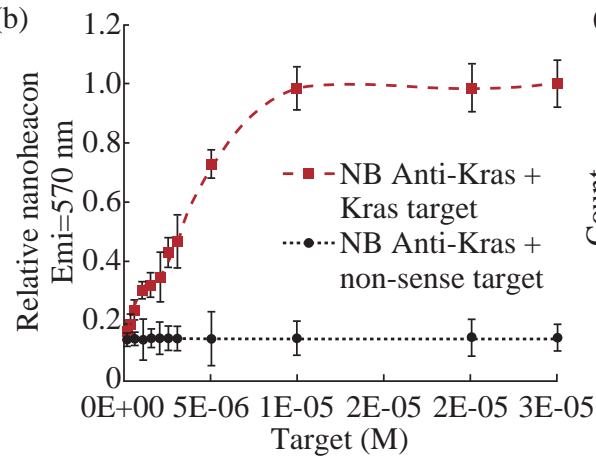

(d) 1.5

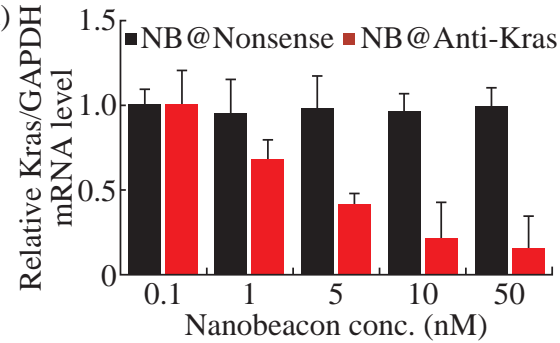

Systemic

administration

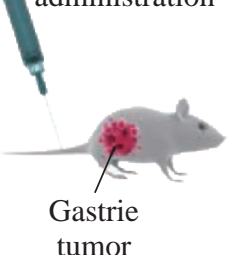

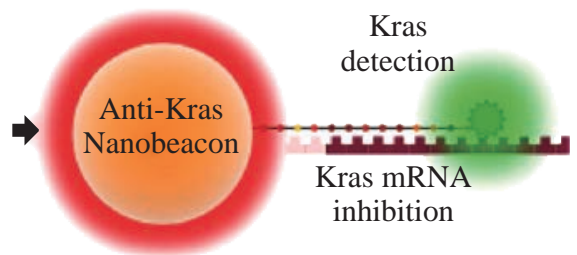

(c)
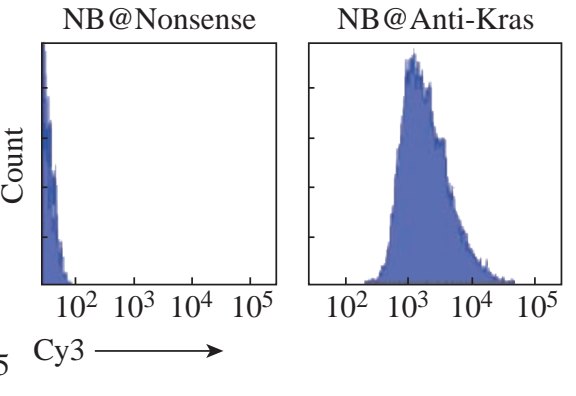

(e)

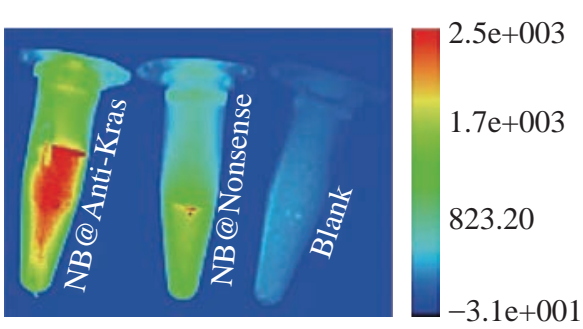

Fig. 5 (a) Gold nanoparticles (AuNPs) functionalized with a dye (Cy3) labeled hairpin-DNA, i.e. gold-nanobeacon (Aunanobeacon), designed to detect, target and inhibit the expression of Kras gene in an in vivo murine gastric cancer model. Under hairpin configuration, proximity to gold nanoparticles leads to fluorescence quenching; hybridization to a complementary target restores fluorescence emission due to the Au-nanobeacons' conformational reorganization that causes the dye and the AuNP to part from each other. The scheme was designed and produced on Adobe Illustrator CC 2014 by J. Conde; (b) Cy3 emission at $570 \mathrm{~nm}$ after hybridization with increasing amounts of complementary and non-complementary ssRNA targets (0 to $\left.3 \times 10^{-5} \mathrm{M}\right)$; (c) Flow cytometry analysis comparing nanobeacon anti-Kras treated cells to nanobeacon nonsense; (d) The silencing effect was expressed as a concentration-dependent decrease in Kras relative expression. Anti-Kras nanobeacons showed potent silencing effects in human gastric cancer cells (MGC-803) with an ED50 between 1 and $5 \mathrm{nM}$; (e) Live imaging images of anti-Kras nanobeacon (previously incubated with K-ras complementary target) compared to nanonsense nanobeacon and a blank tube. Reprinted with permission from Nature Press [23]. 
mRNA) restores fluorescence emission due to the gold nanobeacons' conformational reorganization that causes the fluorophore and the quencher to part from each other, yielding a quantitative response. These molecular beacons have the ability to interlock or hybridize with the target Kras mRNA (blocking specific translation initiation signals of Kras gene) thus inhibiting the translation of the target protein. In addition to designing anti-Kras nanobeacon that detects and inhibits Kras mRNA, a nonsense nanobeacon (that does not hybridize with any target) was developed as an internal control. These bioresponsive gold nanobeacons have a unique and positive effect on metastatic cell colonization in the lungs in a syngeneic and spontaneous gastric cancer mice model. This nanobeacons platform is of high therapeutic potential and serves as a theranostics system of orthotopic gastric tumors but also as anti-metastasis treatment, where nanobeacons show a strong involvement in both local and distant lesions [23].

Another nanoprobe was also developed for targeted imaging and selective therapy of in situ gastric cancer with HER2 overexpression. A multifunctional HER2 monoclonal antibody-conjugated RNase A-associated CdTe quantum dot cluster (HER2-RQDs) nanoprobes was prepared, subcutaneous gastric cancer nude mouse models and in-situ gastric cancer SCID mouse models were established, and were intravenously injected with HER2-RQDs nanoprobes, the biodistribution and therapeutic effects of HER2-RQDs in vivo were evaluated. Results showed that HER2-RQDs nanoprobes could selectively kill gastric cancer MGC803 cells, could target imaging subcutaneous gastric cancer cells at 3 hrs post-injection, and in situ gastric cancer cells at 6 hrs post-injection, and could inhibit the growth of gastric cancer tissues and extended survival time of gastric cancer bearing mouse models, as shown in Fig. 6, the mechanism is closely associated with destroying functional RNAs in cytoplasm by RNase A released from HER2RQDs nanoprobes, preventing protein synthesis and inducing cell apoptosis [24]. This novel HER2-RQDs nanoprobes can be used for in situ gastric cancer genotyping, targeted imaging and selective therapy in the near future.

\section{FMNPs-labeled MSCs for targeted imaging and hyperthermia therapy}

We also prepared FMNPs-labeled human MSCs, and realized targeted imaging and hyperthermia therapy

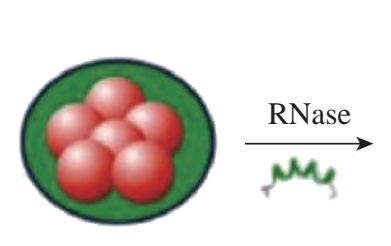

QDs

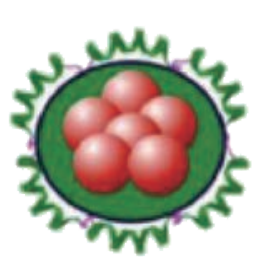

RQDs

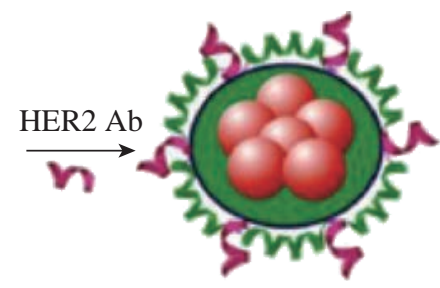

HER2-RQDs

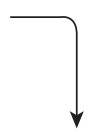

HER2

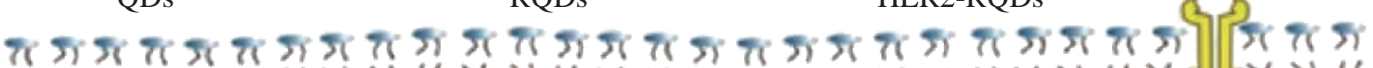

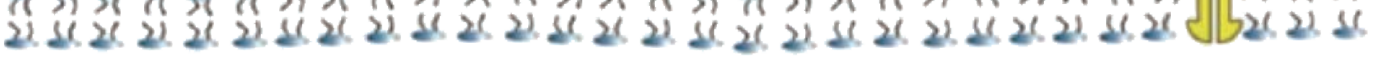

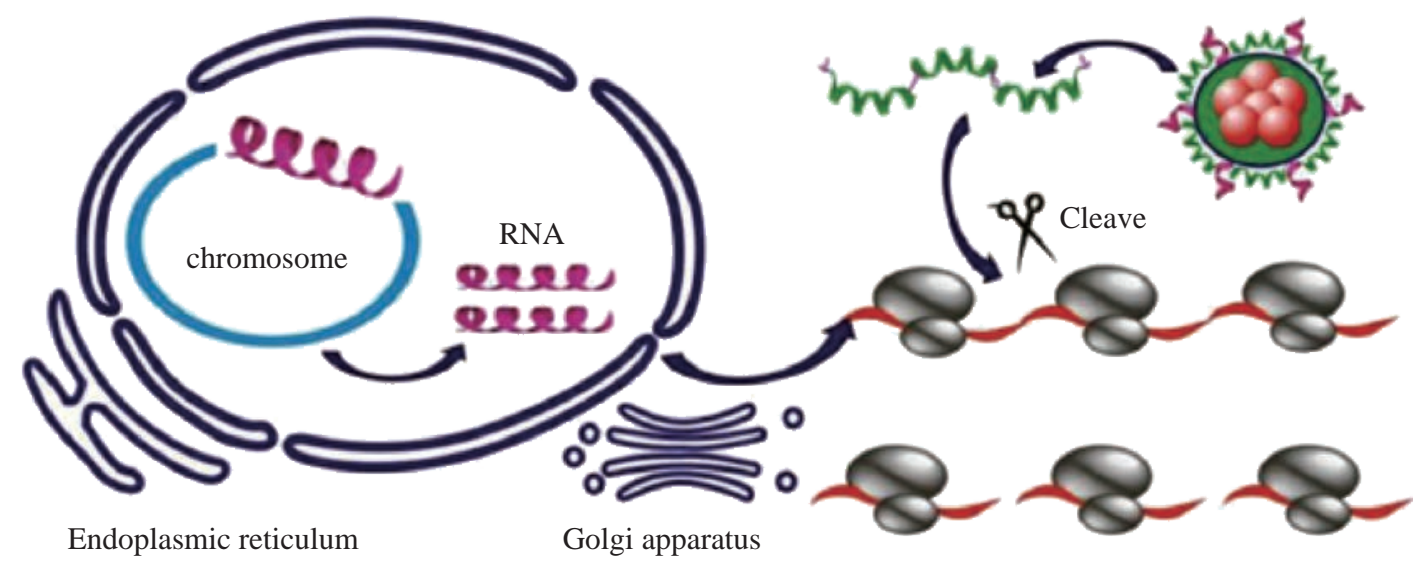

Fig. 6 Therapeutic mechanism of as-prepared nanoprobes: HER2-RQDs nanoprobes could specifically enter into gastric cancer cells, RNase A was released from the HER2-RQDs nanoprobes under the acid environment, which degraded total RNAs inside gastric cancer cells, inhibiting RNA translation and protein synthesis, and finally induced tumor cells' apoptosis. Reprinted with permission from Wiley Press [24]. 
of gastric cancer. We firstly proposed the concept of stem cell nanotechnology, which is an emerging interdisciplinary field, which refers to the application of nanotechnology in stem cell research and development. Although great advances in the field of stem cells have been and being made, several obstacles must be overcome before their therapeutic application can be realized. These include the development of advanced techniques to understand and control functions of micro-environmental signals and novel methods to track and guide transplanted stem cells. The application of nanomaterials and nanotechnology in stem cells research and development exhibits attracting technological prospects, which provide a new chance to solve current problems that stem cells research and development meet $[25,26]$.

Mesenchymal stem cells (MSCs) are multipotent stem cells that can differentiate into a variety of cell types, including: osteoblasts (bone cells), chondrocytes (cartilage cells) and adipocytes (fat cells). MSCs possess immunosuppressive or immunomodulatory properties and have the characteristics of home to the sites of active tumorigenesis. Thus, MSCs can be considered as a candidate cell type for cell-based tissue engineering, cancer therapeutics and regenerative medicine applications. However, the distribution and final fate of MSCs cells inside human body is still not clarified well, which urgently need the novel labeling and in vivo tracking technology.

As shown in Fig. 7, MSCs were characterized. The silica-coated fluorescent super-magnetic nanoparticles (FMNPs) were prepared, were evaluated their effects on mesenchymal stem cells (MSCs), and were used to label MSCs, and then observed the distribution and final sites of the labeled MSCs in vivo in nude models loaded with gastric cancer. Some chemoattractant cytokines were analyzed by ELISA and Western Blotting. As shown in Fig. 8, MSCs were labeled
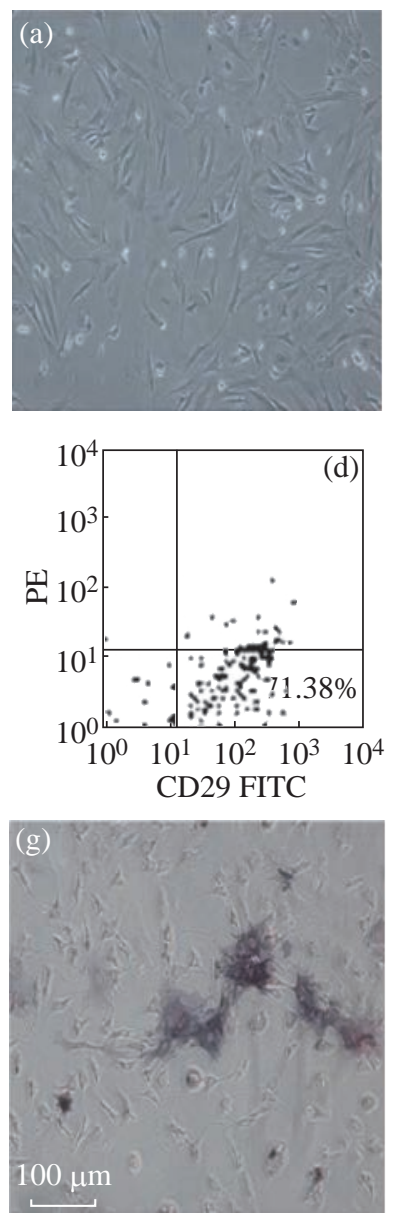
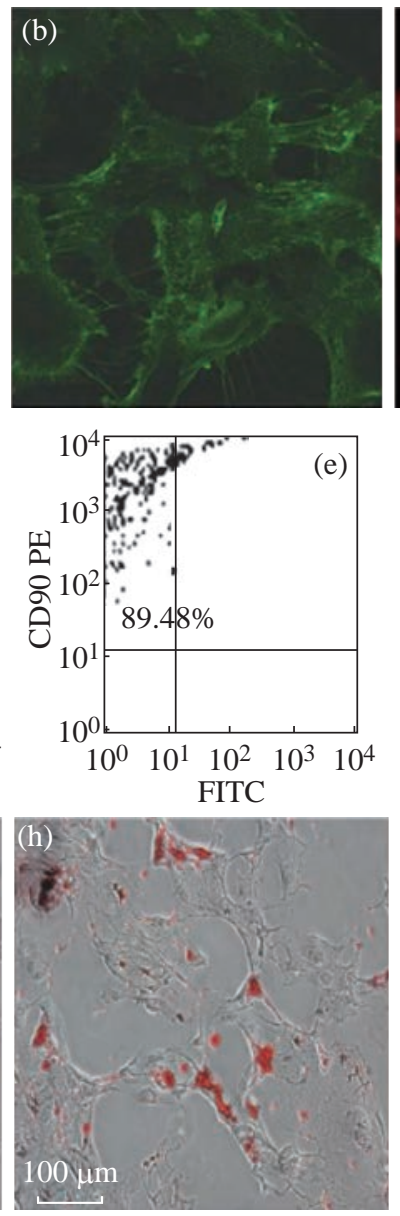
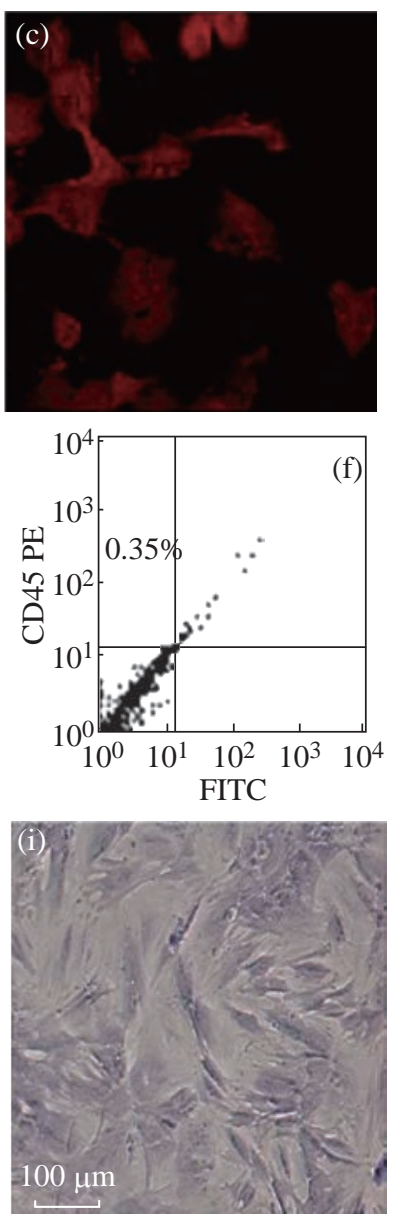

Fig. 7 Morphology and immunophenotypic characterization of MSCs. (a) The fibroblastic morphology of passage 3 MSCs (magnification $=\times 100$ ); (b) MSCs stained with FITC-conjugated CD29 antibody $(\times 200)$; (c) MSCs stained with PE-conjugated CD90 antibody ( $\times 200)$; MSCs analyzed by FACS for the positive expression of (d) CD29, (e) CD90, and (f) negative expression of CD45; (g) Differentiated osteoblasts tested with alkaline phosphatase staining $(\times 100)$; (h) Differentiated adipocytes characterized by Oil Red O staining $(\times 100)$; (i) Differentiated chondrocytes verified by toluidine blue staining $(\times 100)$. Reprinted with permission from Springer Press [27]. 

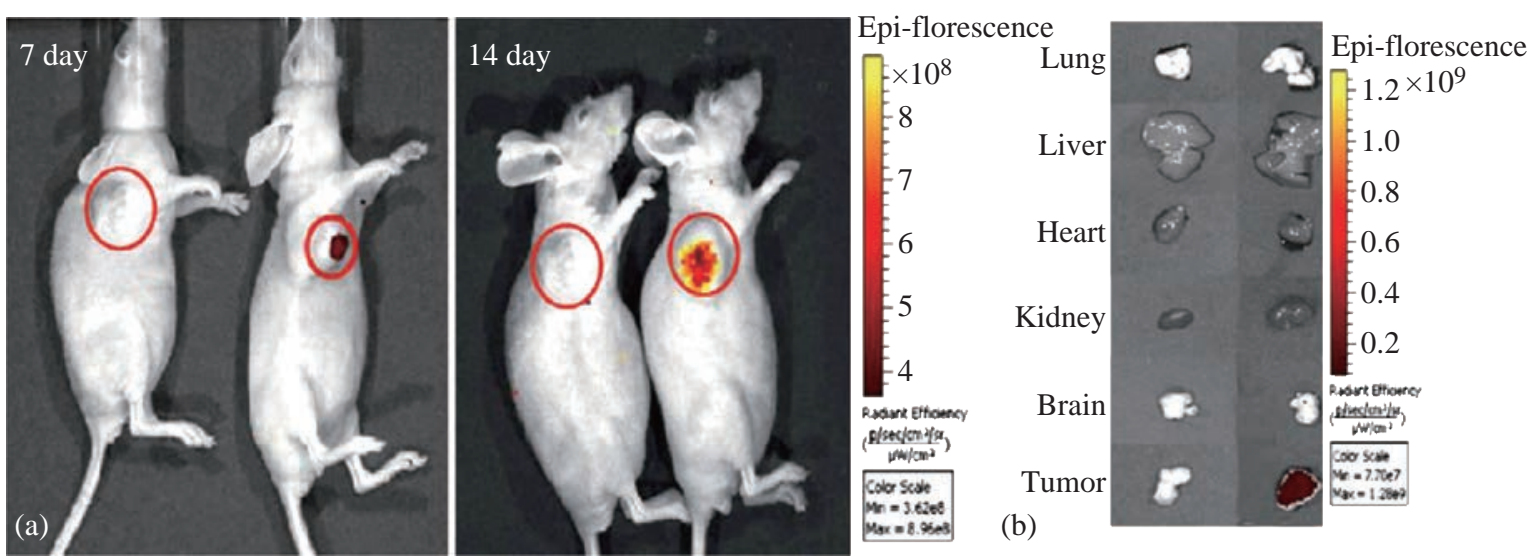

Fig. 8 Fluorescent imaging of FMNP-labeled MSCs targeting gastric cancer cells in vivo. (a) The in vivo fluorescent images show that tumor sites of the mice in the test group had fluorescent signals after post-injection of FMNP-labeled MSCs at 7 and 14 days (right), and tumor sites of the mice in the control group had no fluorescent signal after post-injection of FMNPs at 7 and 14 days (left); (b) The fluorescent imaging of major organs show that no signal was detected in the tumor and organs of the control group (left), and obviously fluorescent signals were detected in the tumor tissues of the test group (right). Reprinted with permission from Springer Press [27].
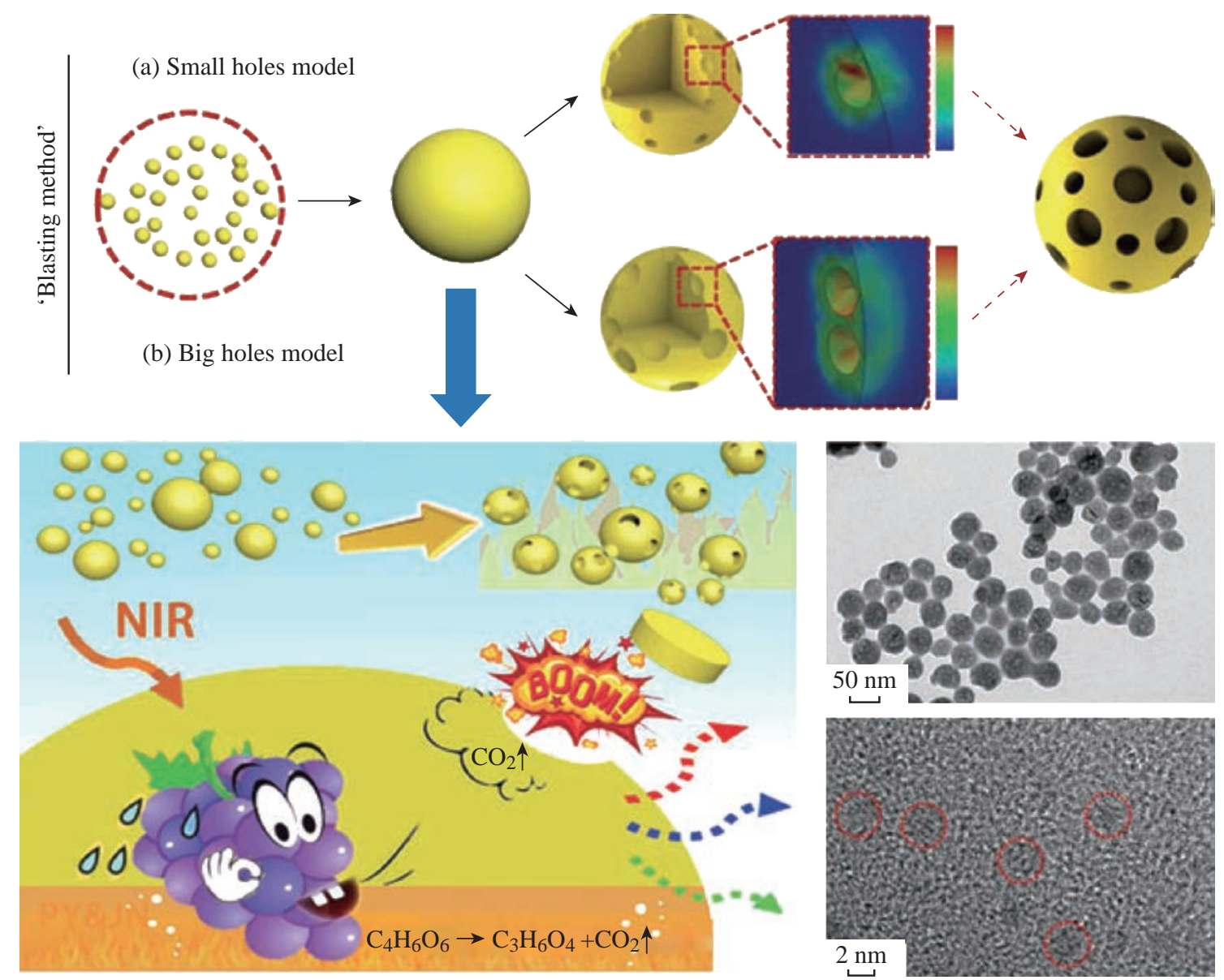

Fig. 9 Illustration of the 'blasting' method for fabricating porous UNs. Reprinted with permission from RSC Press [28].

with FMNPs efficiently and kept stable fluorescent signal and magnetic properties within 14 days, FMNPlabeled MSCs could target and image in vivo gastric cancer cells after being intravenously injected for 14 days, FMNP-labeled MSCs could significantly inhibit the growth of in vivo gastric cancer because of hyperthermia effects, and CCL19/CCR7 and CXCL12/ CXCR4 axis loops may play key roles in the targeting of MSCs to in vivo gastric cancer. This FMNPs-based labeling and tracking technology have great potential 
(I) Vitro CT images of $\mathrm{NaGdF}_{4}$ "nanoclusters"

(a)

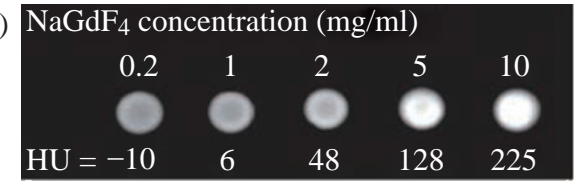

(b)

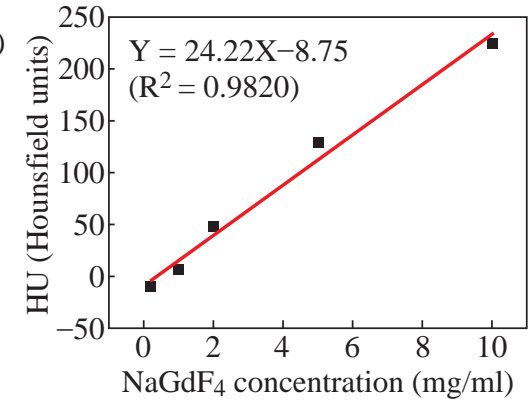

(III) CT images of vivo nude mice
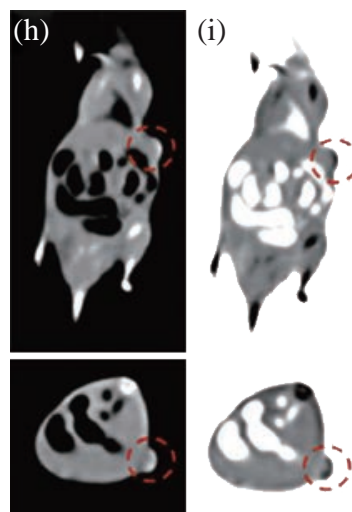

(II) Control group images of vivo nude mice
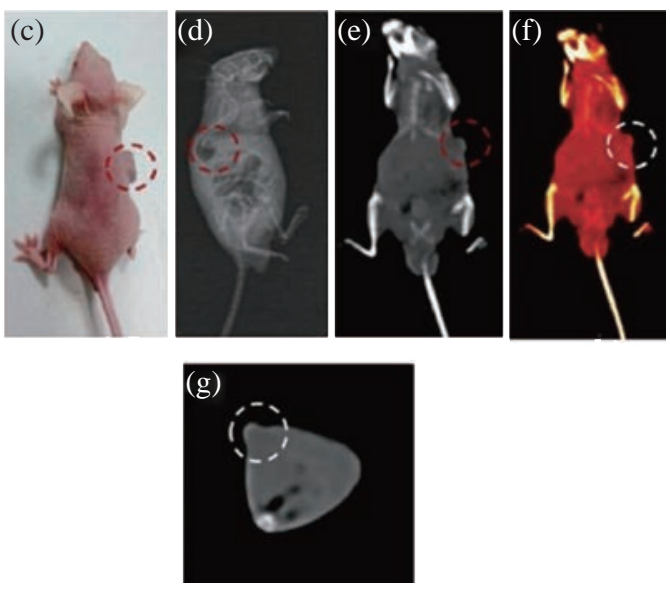

(IV) MRI images of vivo nude mice
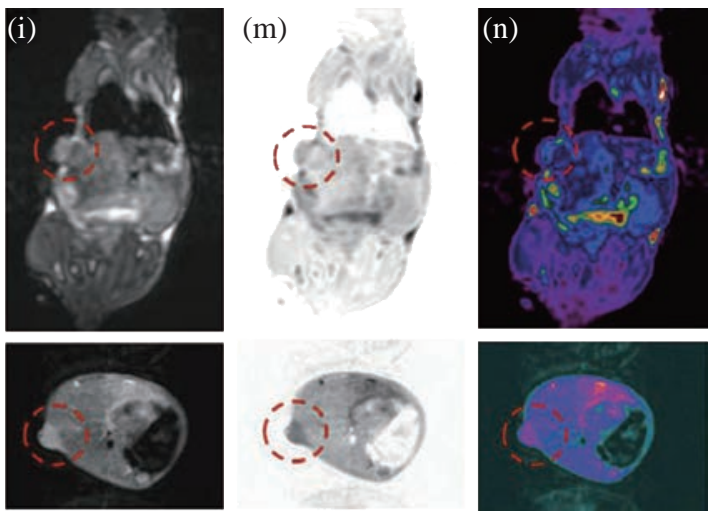

Fig. 10 (I) (a) In vitro CT images of lanthanide-doped NaGdF 4 upconversion "nanoclusters" (sub-5 nm) suspended in aqueous solution; (b) CT attenuation (HU) plot of $\mathrm{NaGdF}_{4}$ changes along with the concentration of each sample from $0.2-10 \mathrm{mg} \mathrm{ml}^{-1}$ to further investigate the CT contrast effect. (II) Images of control group before injection: (c) photograph of the nude mouse model loaded with gastric cancer MGC-803 cells; (d) X-ray image and (e)-(g) CT images of nude mouse as the control group. (III) CT images (h)-(k) and (IV) MRI images (l)-(n) with $\mathrm{NaGdF}_{4}$ upconversion "nanoclusters" through intravenous injection under the function of passive targeting. Reprinted with permission from RSC Press [28].

in applications such as labeling and tracking implanted cells, evaluating cell therapeutic effects, recognizing and mapping early gastric cancer cells [27].

\section{Folic acid-conjugated silica-modified upconversion nanoprobes fortargeted UCL and $\mathrm{CT}$ imaging}

Folic acid-conjugated silica-modified $\mathrm{LaF}_{3}: \mathrm{Yb}, \mathrm{Tm}$ upconversion nanoparticles (UCNPs@SiO ${ }_{2}$-FA) with high La content in single particle were strategically designed and prepared for simultaneous targeting dualmodality imaging of upconversion luminescence (UCL) and X-ray computed tomography (CT) [28]. $\mathrm{LaF}_{3}$ UCNPs were synthesized by a novel oleic acid (OA)/ ionic liquid (IL) two-phase system. Afterwards, folic acid molecule was covalently anchored on the surface of UCNPs with silane coupling agent. The UCNPs@ $\mathrm{SiO}_{2}$-FA exhibits good stability, water dispersibility and solubility, noncytotoxicity, good biocompatibility, highly selective targeting, excellent X-ray attenuation and UCL emission under excitation at $980 \mathrm{~nm}$. In vivo UCL and CT images of mice show the UCNPs@SiO ${ }_{2}-$ FA can be used in targeting dual-modality imaging. These results suggest that the as-prepared nanoprobe is a good candidate with excellent imaging and targeting ability for targeting dual-modality imaging of UCL and CT.

Multimodal contrast agents based folic acidconjugated silica-modified $\mathrm{LaF}_{3}: \mathrm{Yb}, \mathrm{Tm}$ upconversion nanoparticles (UCNPs@SiO ${ }_{2}$-FA) with high La content in single particle were strategically designed and developed. We have demonstrated that UCNPs@ $\mathrm{SiO}_{2}$-FA is a good candidate with excellent imaging and targeting ability for UCL imaging and CT imaging invivo. The prepared UCNPs@SiO ${ }_{2}-$ FA exhibits good stability, water dispersibility and solubility, noncytotoxicity, good biocompatibility, highly selective targeting, excellent $\mathrm{X}$-ray attenuation 


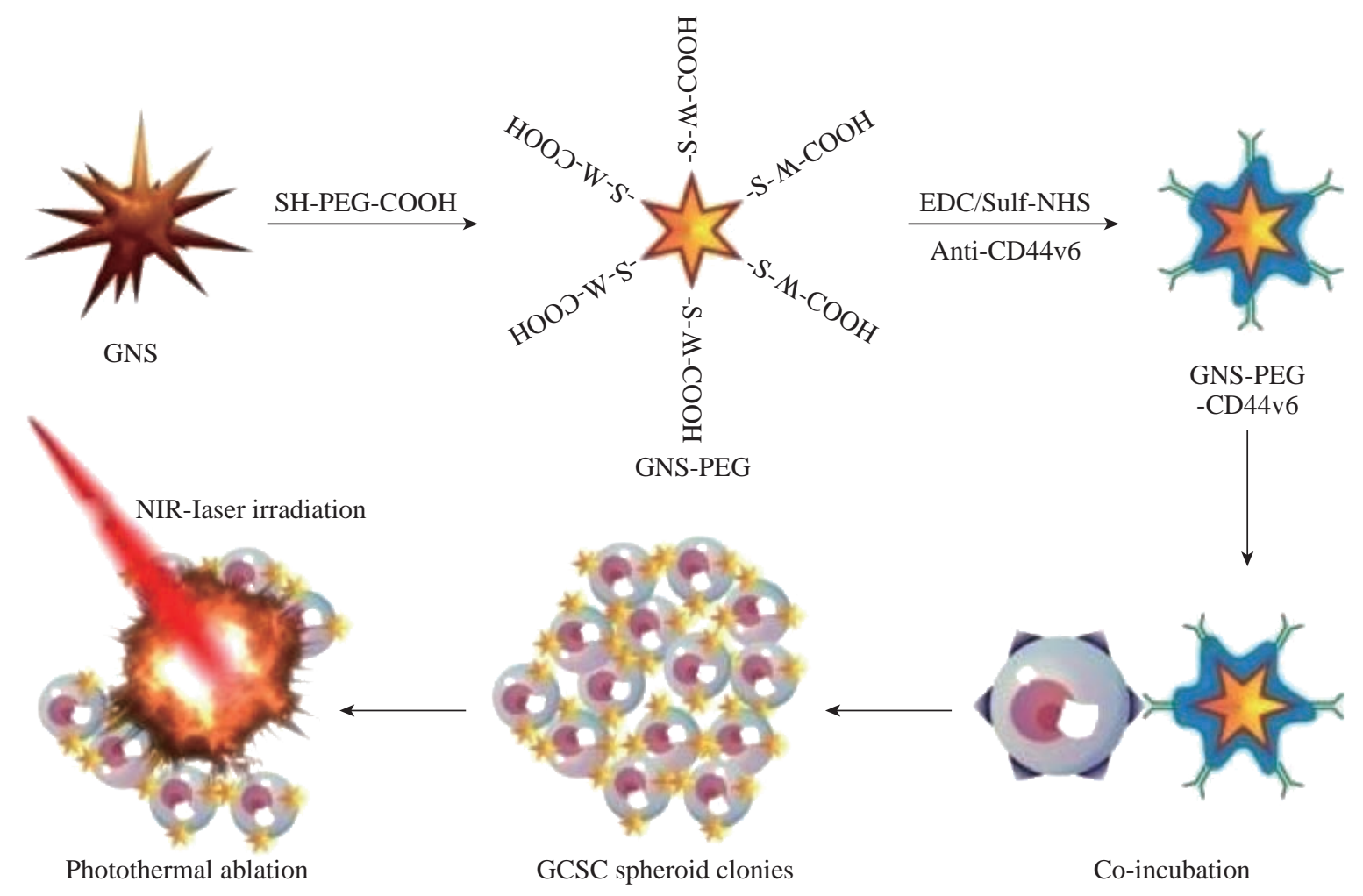

Fig. 11 Schematic illustration of the GNS-PEG-CD44v6 nanocomplex synthesis process and the mechanism of GCSC targeted PTT. Reprinted with permission from IVY press [30].

and UCL emission under excitation at $980 \mathrm{~nm}$. Our results indicated that the synthesized UCNPs@ $\mathrm{SiO}_{2}$-FA is effective for simultaneous targeting dualmodality imaging of UCL and CT. It could bring novel opportunities to the next generation of theranostic nanoprobes for simultaneous diagnosis and treatment in vivo.

We also developed a novel synthesis method of UCPs, hydrothermal system in which an anion induces the phase transition process to give simultaneous control over the size, morphology, phase and emission properties. We first confirm that the crystal cell oriented-rotation driven by an anion in a hydrothermal system promoted the phase transition, and the energy zones figure of the phase transition from cubic to hexagonal structure has been figured out. We have successfully applied the structural mechanics finite element calculations to validate the reaction process. We have also demonstrated that porous UNs can be rationally tuned in size (down to fifteen nanometers), phase (cubic or hexagonal) and emission properties at precisely defined conditions, and were effective for in vitro and in vivo CT imaging [29].

CD44v6 antibody-conjugated gold nanostars for photoacoustic imaging and photothermal

\section{therapy of gastric cancer stem cells}

Developing safe and effective nanoprobes for targeted imaging and selective therapy of gastric cancer stem cells (GCSCs) has become one of the most promising anticancer strategies [30-34]. Herein, gold nanostars-based PEGylated multifunctional nanoprobes were prepared with conjugated CD44v6 monoclonal antibodies (CD44v6-GNS) as the targeting ligands. It was observed that the prepared nanoprobes had high affinity towards GCSC spheroid colonies and destroyed them completely with a low power density upon near-infrared (NIR) laser treatment $(790 \mathrm{~nm}$, $1.5 \mathrm{~W} / \mathrm{cm}^{2}, 5 \mathrm{~min}$ ) in vitro experiment. Orthotopic and subcutaneous xenografted nude mice models of human gastric cancer were established. Subsequently, biodistribution and photothermal therapeutic effects after being intravenously injected with the prepared nanoprobes were assessed. Photoacoustic imaging revealed that CD44v6-GNS nanoprobes could target the gastric cancer vascular system actively at 4 hrs post-injection, while the probes inhibited tumor growth remarkably upon NIR laser irradiation, and even extended survivability of the gastric cancer-bearing mice. The CD44v6-GNS nanoprobes exhibited great potential for applications of gastric cancer targeted 

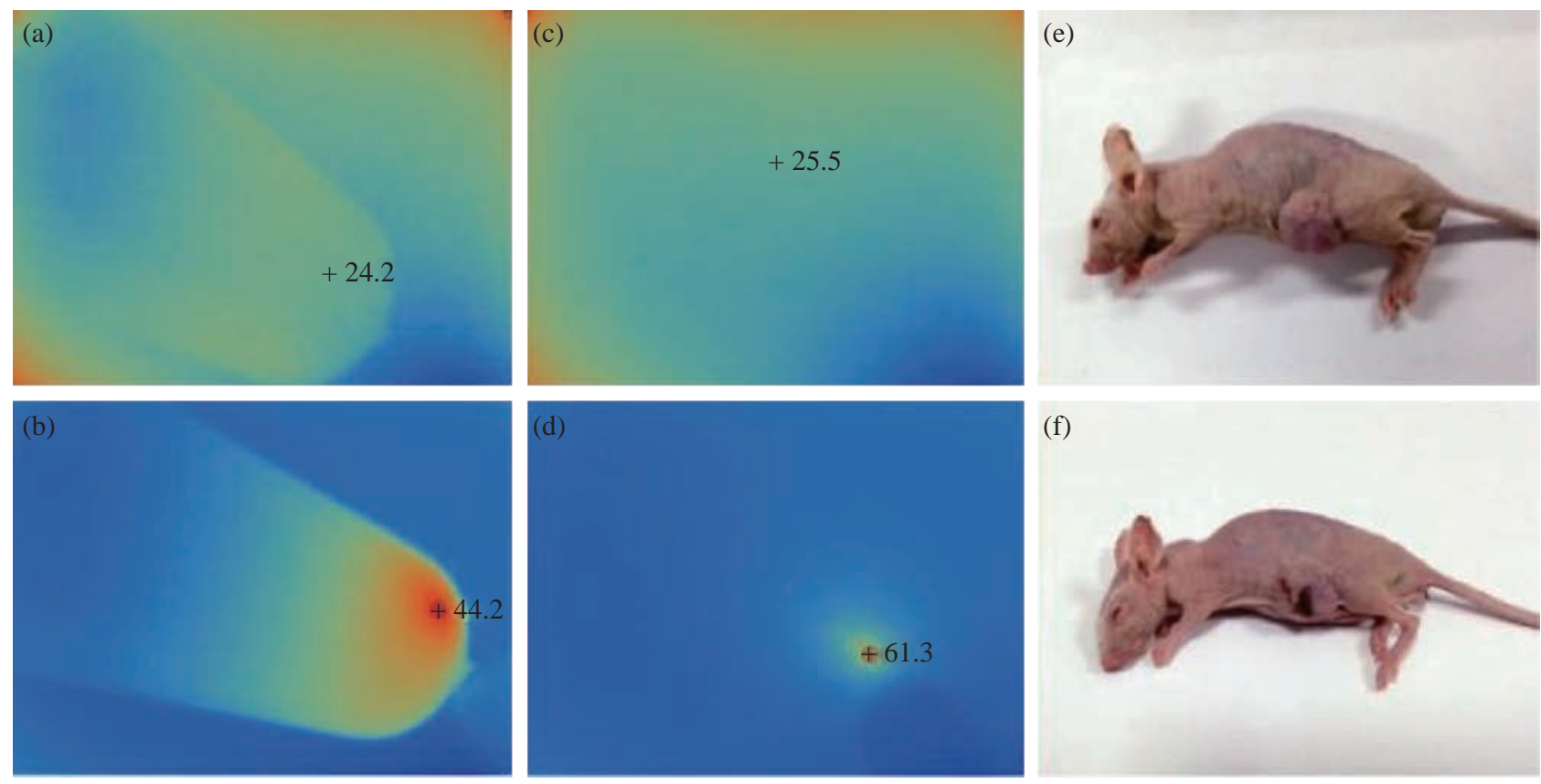

(f)
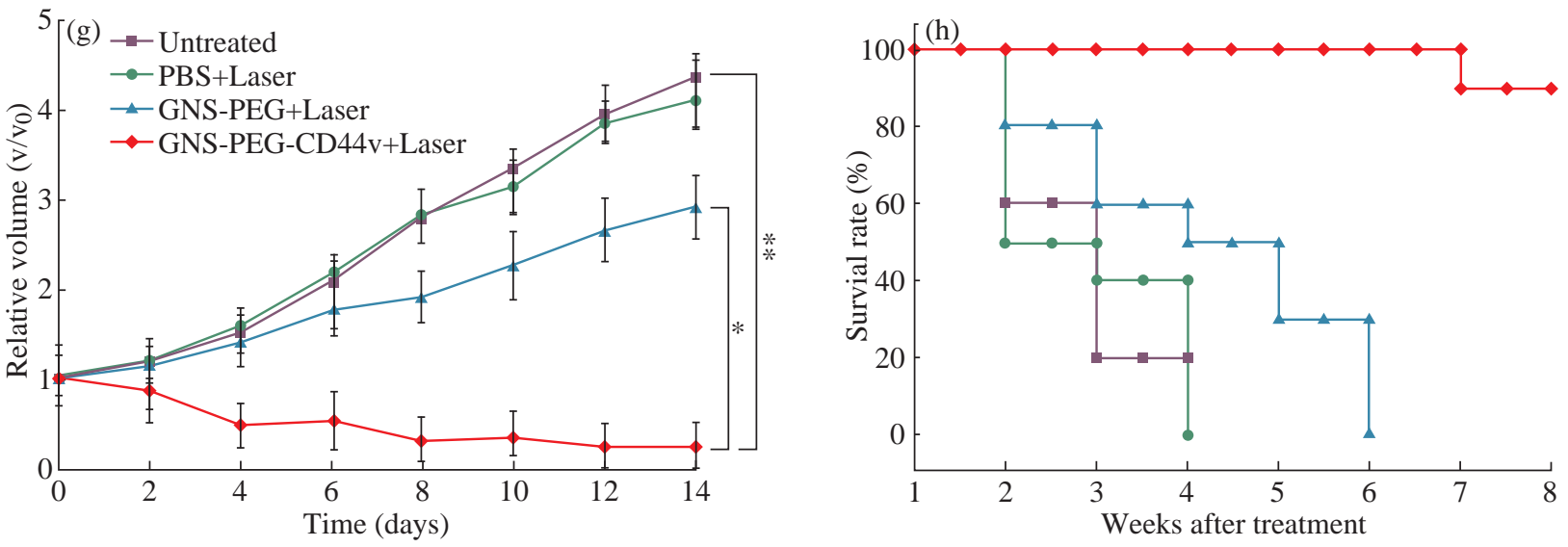

Fig. 12 Infrared microscopic imaging: deionized water (a) and GNS (b) in a tube upon NIR laser irradiation $\left(790 \mathrm{~nm}, 0.3 \mathrm{~W} / \mathrm{cm}^{2}, 3\right.$ $\mathrm{min}$ ); Subcutaneous tumor of GC without (c) and with (d) injecftion of GNS-PEG-CD44v6 upon NIR laser irradiation (790 nm, 1.5 $\mathrm{W} / \mathrm{cm}^{2}, 3 \mathrm{~min}$ ). The nude mouse of GC subcutaneous xenograft, injected with nanoparticles before (e) and after (f) laser irradiation treatment (790 nm, $\left.1.5 \mathrm{~W} / \mathrm{cm}^{2}, 3 \mathrm{~min}\right)$; (g) Tumor growth curves of four groups after treatment with GNS-PEG-CD44v6, GNS-PEG and PBS respectively upon NIR laser irradiation $\left(790 \mathrm{~nm}, 0.8 \mathrm{~W} / \mathrm{cm}^{2}, 5 \mathrm{~min}\right)$; and the untreated control group. Error bars represent standard deviation ( $\mathrm{n}=10$ /group). ${ }^{*} \mathrm{p}<0.05,{ }^{*} \mathrm{p}<0.01$, (Student's $\mathrm{t}$ test); (h) Survival rate of GC tumor-bearing mice within eight weeks after treatment. Reprinted with permission from IVY Press [30].

imaging and photothermal therapy in the near future $[35,36]$.

\section{Folic acid/ce6 conjugated gold nanoclusters for NIR fluorescent imaging andphotodynamic therapy}

Gold nanoclusters (GNCs) attract increasing attention due to their potential applications in sensing, catalysis, optoelectronics, and biomedicine. Herein, the formation of highly fluorescent glutathione (GSH)capped GNCs is achieved through the delicate control of the reduction kinetics and thermo-dynamic selection of the $\mathrm{Au}(\mathrm{I})-\mathrm{SG}$ complexes. Furthermore, the GNCs- based nanoprobes are developed by the covalent coupling folic acid (FA) and PEG (polyethylene glycol) on the surface of GNCs directly, followed by trapping photosensitizer (chlorine6, Ce6) within PEG networks and attaching to the GNCs surface. The fabricated nanoprobes (Ce6@GNCs-PEG 2KFA) possess a uniform particle size (hydrodynamic diameter $=6.1 \pm 1.2 \mathrm{~nm}$ ), without affecting the yield of singlet oxygen of the trapped Ce6. In vitro studies show the enhanced cellular uptake and satisfactory photodynamic therapy (PDT) effectiveness toward MGC-803 cells when compared with free Ce6. The biodistribution and excretion pathway studies of the 


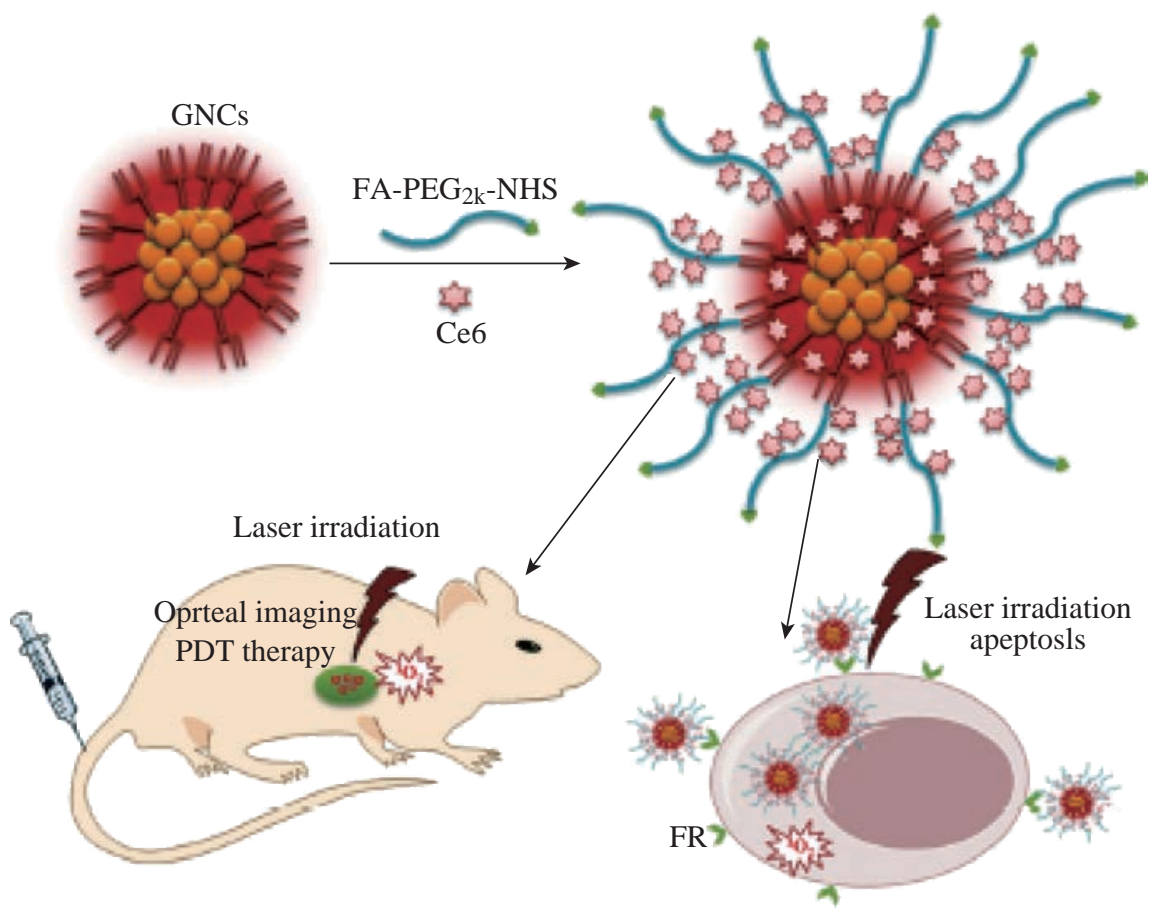

Fig. 13 Schematic illustration of the preparation of the GNCs-based nanoprobes and their applications in vitro and in vivo. Reprinted with permission from Wiley Press [37].

(a)

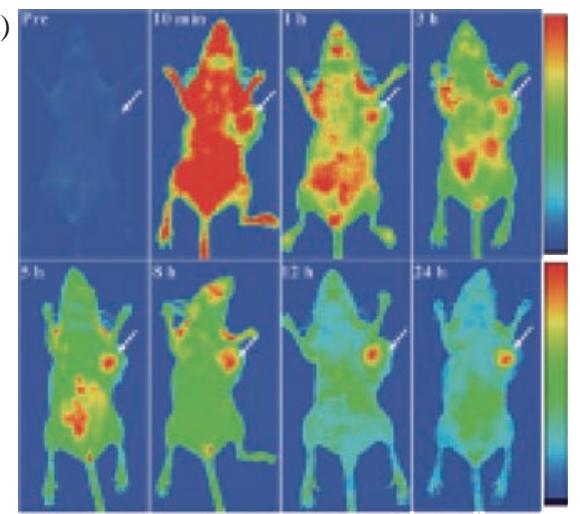

(c) 250

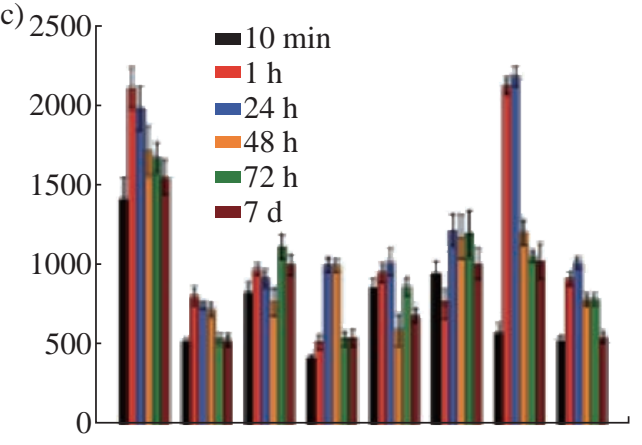

(b)

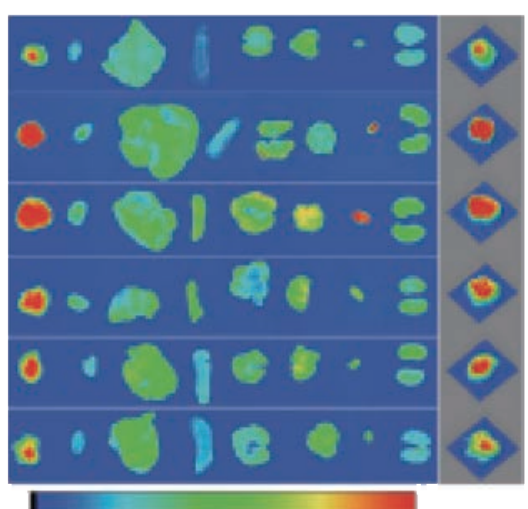

(d)

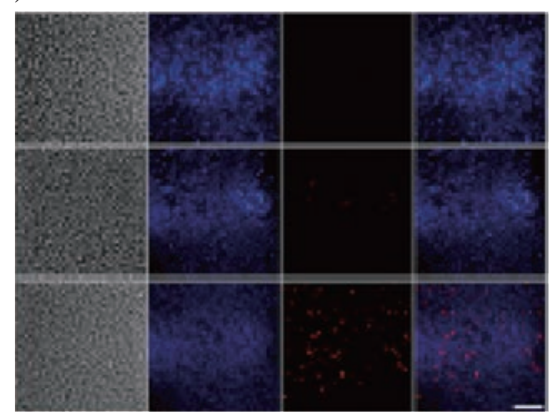

Fig. 14 (a) In vivo fluorescence imaging of MGC-803 tumor-bearing mouse after intravenous injection of Ce6@GNCs-PEG 2KFA over a period of 24 hrs; (b) Representative ex vivo fluorescence images of major organs and tumors of mice after different postinjection times. T (tumor), $\mathrm{H}$ (heart), Li (liver), S (spleen), Lu (lung), Br (brain), Bl (bladder), K (kidney). The right columns were the corresponding 3D surface plot images of pixel intensities obtained from the tumor regions using NIH Image J software; (c) Quantitative biodistribution of Ce6@GNCs-PEG 2K -FA in different organs and tumors based on ROIs analysis of the average fluorescence intensity from the tumors and normal tissues (3 mice per group); (d) Representative fluorescence images of tumor cryosections $(10 \mu \mathrm{m})$. Images presented are bright-field, blue fluorescence (DAPI), red fluorescence (>590 nm), and merged (overlap) images. Scale bar, $100 \mu \mathrm{m}$. Reprinted with permission from Wiley Press [37]. 
(a)

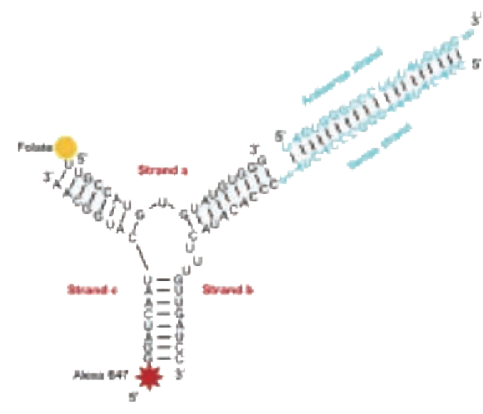

(b)

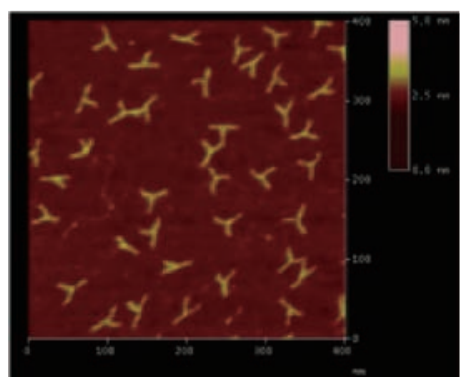

Extended 3WJ RNA Nanoparticle
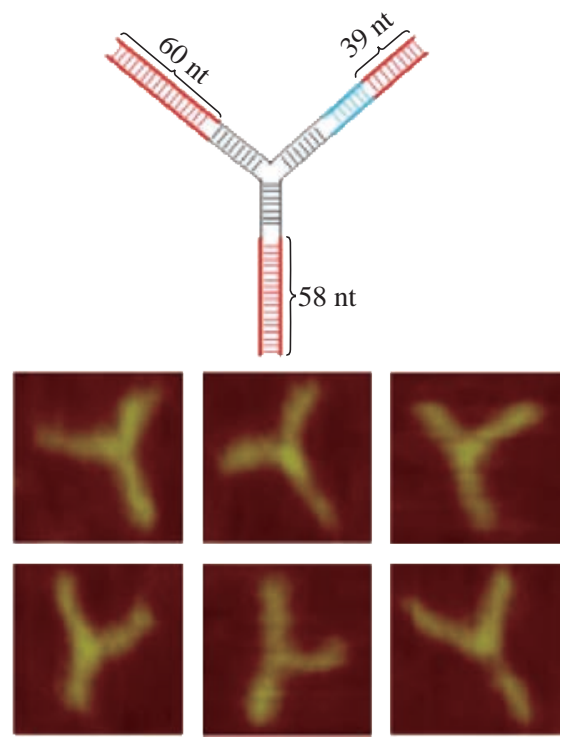

Fig. 15 (a) Design of the RNA nanoparticles. Left is the one use in animal trial. Right is the extended one to prepare the AFM images; (b) AFM image of extended 3WJ RNA nanoparticles. The RNA complex in left of a is estimated to be around $10 \mathrm{~nm}$. Due to convolution of the tip size $(5 \sim 10 \mathrm{~nm}$ in diameter) in AFM images, features close to the size of the tip cannot be resolved. To characterize the structure of the RNA constructs, the 3WJ nanoparticles were extended by 39-60 base-pairs (in red color), which is within the persistence length of dsRNA and will not affect the 3WJ folding as described before, to generate the AFM image as shown. Reprinted with permission from Nature Press [43].

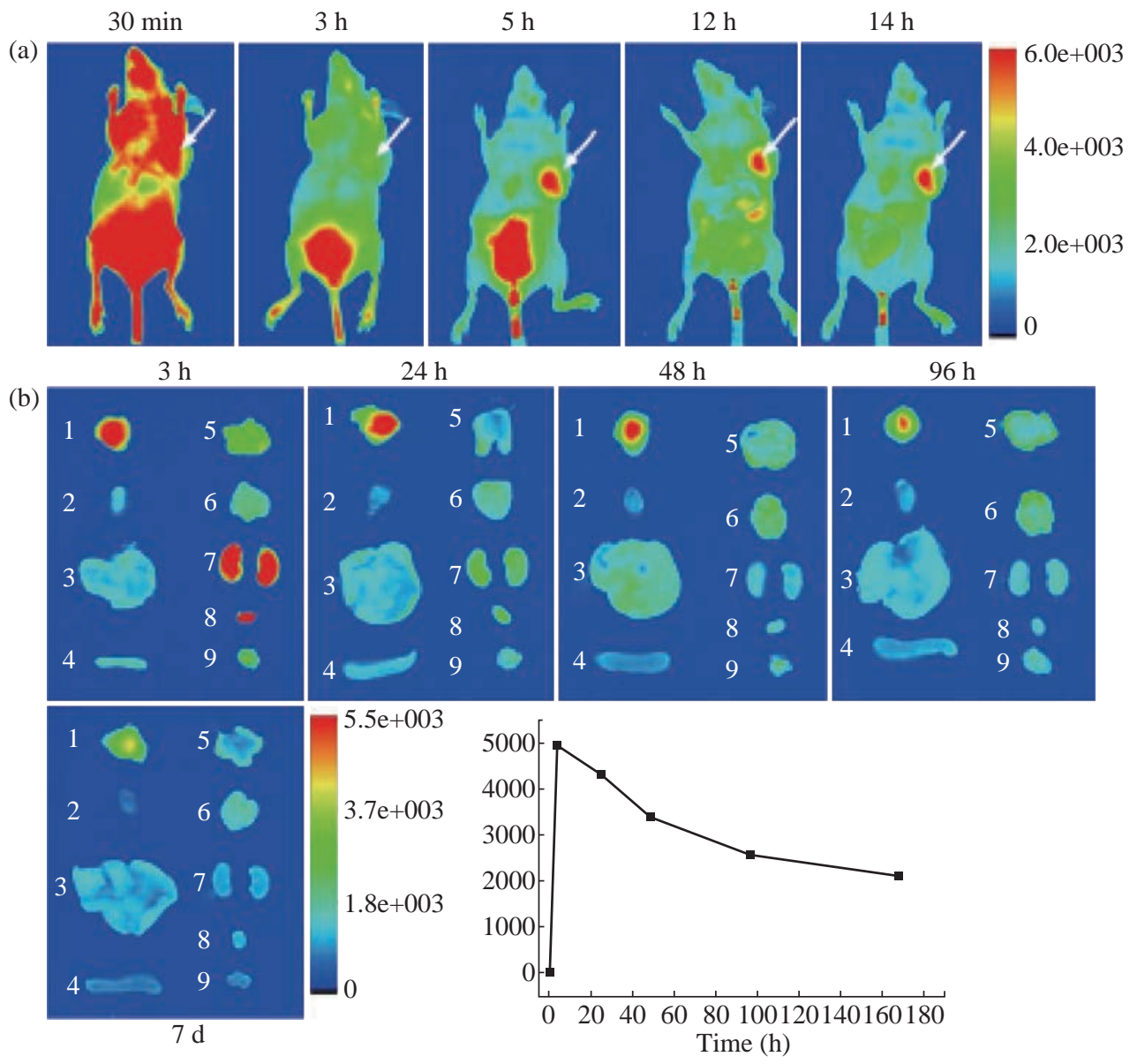

Fig. 16 (a) Representative in vivo fluorescence images of MGC803-tumour-bearing mouse after iv-injected with FA-AlexaFluor647labeled pRNA nanoparticle. The tumor areas are indicated with arrows; (b) Representative ex vivo images of tumours and organs. Labels: 1, tumor; 2, heart; 3, liver; 4, spleen; 5, lung; 6, Stomach; 7, kidneys; 8, bladder; 9, muscle; (c) The average fluorescence intensities from the tumor areas of post-injection. Reprinted with permission from Nature Press [43]. 


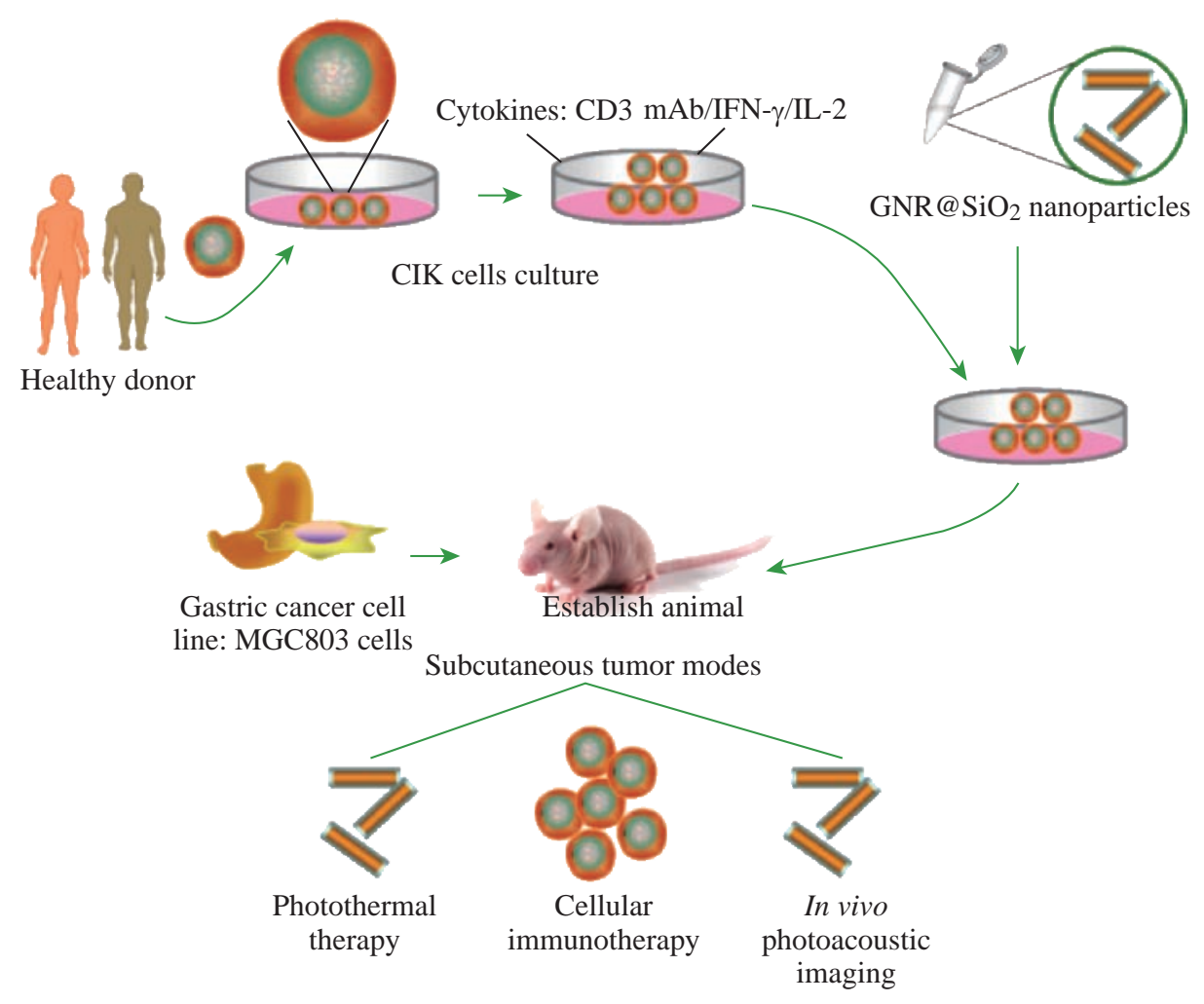

Fig. 17 Schematic illustration of CIK cells with Au nanorods for targeted imaging and immunological cell therapy. Reprinted with permission from Springer Press [45].

nanoprobes in MGC-803 tumor-bearing nude mice reveal their superior penetration and retention behavior in tumors, while the preserved features of renal clearance and stealthy to reticuloendothelial system are mainly attributed to the small hydrodynamic diameters and the FA-capped PEGylated ligands. The enhanced PDT efficacy and the nontoxicity to mice provide an exciting new nano-platform with promising clinical translational potential [37-39].

\section{RNA nanoparticles for targeted imaging and SiRNA therapy of gastric cancer}

In recent years, there have been several new nanodelivery systems with different materials and physicchemical properties to be developed. However, effective strategies to block tumor progression and prevent metastasis are still very few, there exist several challenges including specific cancer targeting, tissue penetration, intracellular delivery, toxicities and side effects due to organ accumulation, nonspecific cell entry, particle heterogeneity, aggregation, dissociation due to dilution after systemic injection, and unfavorable pharmacological profiles.In recent years, RNA nanotechnology, as an emerging nanoplatform, has shown great prospect in clinical theranostic applications. RNA nanoparticles can be fabricated with precise control of shape, size and stoichiometry, as demonstrated by the packaging RNA (pRNA) of the bacteriophage phi29DNApackaging motor, which forms dimmers, trimers, and hexamers, via hand-in-hand interactions of the interlocking loops $[40,41]$. The pRNA contains an ultra-stable three-way junction (3WJ) motif, which can be assembled from three short fragments with extremely high affinity. Recently Prof. Peixuan Guo's group has obtained the crystal structure of the pRNA-3WJ motif and a variety of therapeutic RNA nanoparticles using the pRNA3WJ and pRNA-X motifs as scaffolds have been constructed. The pRNA-3WJ nanoparticles display thermodynamically stable properties, including high melting temperature with low free energy, resistance to denaturation in $8 \mathrm{M}$ urea and resistance to dissociation at very low concentrations in the blood. Boiling resistant RNA nanoparticles with controllable shapes and defined stoichiometry have recently been reported. Various functional groups, such as imaging agents; targeting ligands, such as receptor binding aptamers; and therapeutic modules, such as siRNA, miRNA or ribozymes can be integrated into the $3 \mathrm{WJ}$ scaffold without affecting the folding and functionality of 
the core motif and incorporated functional moieties. Upon 2'-Fluoro (2'-F) modifications of Uracil (U) and Cytosine (C) nucleotides, the RNA nanoparticles become resistant to RNase degradation with enhanced in vivo half-life while retaining authentic functions of the incorporated modules. Furthermore, the pRNA nanoparticles are non-toxic, non-immunogenic, and display favorable biodistribution and pharmacokinetic profiles in mice. These favorable findings prompted the use of this novel platform for the treatment of stomach cancer, which is one of the challenging tasks in clinical oncology.

Such targeted delivery systems need a ligandreceptor pair that is specifically existed in cancer cells. Many, but not all, cancer cells, including stomach, ovary, lung, breast, kidney, endometrium, colon and hematopoietic cells, over-expressed folate receptors (FRs) than normal cells for high uptake of folate, since folate is essential component during DNA replication and methylation in highly proliferating cells. Folic acid (FA), a synthetic oxidized form of folate, has been widely used as a ligand conjugate in various cancer targeting materials. BRCAA1 (breast cancer-associated antigen 1, AF208045) has been confirmed to exhibit over-expression in breast cancer and gastric cancer, and no or lower expression in normal gastric mucosa and normal breast tissues. Our previous studies have demonstrated that gastric cancer MGC803 cells were transfected with constructed plasmids of shRNABRCAA1, the cell growth was greatly inhibited and the rate of cell apoptosis was significantly higher than those of untransfected group and mock plasmid transfected group. We also screened out a new antigen epitope SSKKQKRSHK, and also screened out matched two monoclonal antibody cell lines, and successfully prepared monoclonal antibody conjugated fluorescent magnetic nanoparticles, and realized the targeted imaging and hyperthermal therapy of in vivo gastric cancer. Therefore, the BRCAA1 gene is a potential therapeutic target for gastric cancer.

My group cooperated with Prof. Peixuan Guo's group, adopted an innovative RNA nanotechnology approach to overcome some of the aforementioned challenges, and report for the first time a new strategy to target and deliver therapeutic BRCAA1 siRNA to in vivo stomach cancer tissues using FA-conjugated pRNA-3WJ nanoparticles. Our objective is to construct multi-functional, thermodynamically and chemically stable RNA nanoparticles harboring sequences that allow specific binding to stomach cancer specific cell surface antigens or receptors resulting in the internalization of RNA nanoparticles into target cells and delivery of the siRNA, miRNA, and drugs for attaining synergistic effects for the treatment of stomach cancer, we also investigated the effects of prepared RNA nanoparticles on the regression of gastric cancer tissues in vivo, and potential molecular mechanism, with the aim of laying foundation for further clinical application in near future [42, 43].

RNA interference via the use of small-interfering RNA (siRNA) is a powerful and useful tool to block gene function through sequence-specific posttranscriptional gene silencing, playing an important role in the down-regulation of gene expression. siRNAs can be transfected into mammalian cells by a variety of methods that influence the strength and duration of the silencing response, which in turn is affected by the amount of siRNA effectively delivered and by the potential of each siRNA to suppress its target. Nevertheless, naked siRNAs show extremely short half-lives due to RNase activity, poor chemical stability, and dissociation from the vector. In fact, the major obstacle to clinical application is the uncertainty about how to deliver siRNA with maximal therapeutic impact [44].

\section{Human CIK cells loaded with Au nanorods as a theranostic platform for targeted photoacoustic imaging and enhanced immunotherapy and photothermal therapy}

How to realize targeted photoacoustic imaging, enhanced immunotherapy, and photothermal therapy of gastric cancer has become a great challenge. Herein, we reported for the first time that human cytokineinduced killer cells (CIK) loaded with gold nanorods were used for targeted photoacoustic imaging, enhanced immunotherapy, and photothermal therapy of gastric cancer. Silica-modified gold nanorods were prepared; then incubated with human cytokine-induced killer cells (CIK), resultant human CIK cells loaded with Au nanorods were evaluated for their cytotoxicity, targeted ability of gastric cancer in vitro and in vivo, immunotherapy, and photothermal therapy efficacy. In vitro cell experiment shows that human CIK cells labeled with gold nanorods actively target gastric cancer MGC803 cells, inhibit growth of MGC803 cells by inducing cell apoptosis, and kill MGC803 cells under low power density near-infrared (NIR) laser treatment (808-nm continuous wave laser, 1.5 $\mathrm{W} / \mathrm{cm}^{2}, 3 \mathrm{~min}$ ). In vivo experiment results showed 
that human CIK cells labeled with gold nanorods could target actively and image subcutaneous gastric cancer vessels via photoacoustic imaging at 4 hrs postinjection, could enhance immunotherapy efficacy by up-regulating cytokines such as IL-1, IL-12, IL-2, IL-4, IL-17, and IFN-gamma, and kill gastric cancer tissues by photothermal therapy via direct injection into tumor site under near-infrared (NIR) laser irradiation [45]. High-performance human CIK cells labeled with Au nanorods are a good novel theranostic platform to exhibit great potential in applications such as tumor-targeted photoacoustic imaging, enhanced immunotherapy, and photothermal therapy in the near future.

\section{Clinical Translational Prospects of Theranostic Technologies of Gastric Cancer}

Gastric cancer is one kind of the most common tumors, to realize its prewarning and early theransotics has great clinical requirement. For example, SNP arrays based on magnetic nanoparticles has been explored for clinical application. Four SNP loci (rs2279115, rs804270, rs909253 and rs3765524) have been confirmed to be associatied with gastric cancer risk, which may be used to predict gastric cancer risk.

The VOC biomarkers and breath analysis sensor were confirmed to be able not only to screen early gastric cancer, but also to distinguish EGC from AGC, which has finished part clinical trials. It is possible that breath analysis based on nano-sensor will become a fourth routine examination technique for early screening and stage-determination of gastric cancer in near future.

Some nano-devices such as disposable easy-touse electrochemical microfluidic chip combined with multiple antibodies against six kinds of biomarkers can be used for clinical examination, which can be quickly translated into clinical application because of its mature technology. In addition, CTC microfluidic system has been developed for CTCs capture counts and recollection. Clinical specimen test has confirmed this system is effective for CTCs isolation and recollection, which will be quickly translated into clinical application.

Regarding in vivo nanotheransotic probes, their clinical translation still needs a lot of work. There have been more than several ten thousand publications regarding nanoprobes in the last decade, and very few of the assessed nanoprobes progressed from bench into bedside. According to Drolet and Lorenzi's 'continuum' theory, when a new basic science discovery transfers into proposed human application, proven clinical application, clinical practice and finally raises public health impact, there are three "translation chasm" gaps that must be bridged. These gaps are human (T1), clinical trials (T2) and translation to practice (T3). Although there have been very promising applications of nanoprobes in many areas, they are still at an early stage of development on the way to reaching the bedside; in other words, they are mostly in the T1 stage, although they may come into clinical trials.

Up to date, there are several nanoprobes to enter into clinical trials or obtain FDA permission. For example, in 2011, a clinical trial of insulin-coated AuNPs was approved by Midatech Ltd (UK). In addition, AuroShell $®$ (Au@silica nanoshells) from Nanospectra (Nanospectra Bisciences, TX,USA), which is used for thermotherapy, was approved by the FDA in 2012. This has been proven to be effective at an earlier stage in a murine glioma model and in the ablation of prostate cancer in a canine model. Another example is from McNeil's laboratory; after their reports of the biodistribution, immune responseand interactions of AuNPs with human blood, they used AuNPs as a drug-delivery system in order to impair the toxicity of TNF- $\alpha$. It was confirmed to be safe to inject up to three times the amount that had been lethal with previous versions. The modified drug Aurimune ${ }^{\mathrm{TM}}$ (CYT-6091) from Cytlmmune Sciences (MD, USA) is a TNF $\alpha$ bound, PEGylated colloidal AuNP. Aurimmune has passed Phase I clinical trial and is entering Phase II trial. It was reported that the systemic TNF toxicity of Aurimune was decreased dramatically compared with TNF alone (For example, the fever side effect was managed) and the target effect was realized. Another product, AuriTol ${ }^{\mathrm{TM}}$ (CYT-21001), also from Cytlmmune Sciences, has reached the preclinical stage [46-50].

An example of a fast, in vitro, Au-based biomedical application is the FDA-approved Verigene ${ }^{\circledR}$ detector device (Nanosphere, IL, USA), which utilizes oligonucleotide-coated AuNPs in order to capture 
DNA. The Verigene system enables clinicians to rapidly identify and treat the bacteria and viruses that are responsible for some of the most complicated, costly and fatal infectious diseases [51].

Although some nanoprobes went through the clinical trials, and obtained FDA permission to enter into clinical application, inorganic nanoparticles-based multifunctional nanoprobes remain in great dispute for clinical application, and the reasons are summaried as being unable to degrade in vivo, long- term leftover in body, the further toxicological mechanism is not clarified well. Development of targeting, safe, high efficient nanoprobes has become a great challenge. Only to solve the biosafety of nanoprobes, multifunctional nanoprbes will be possible to enter into clinical application in near future.

\section{Conclusion Remarks}

In summary, gastric cancer is one kind of the most common tumors worldwide, and how to realize theranostics of early gastric cancer is a great challenge. Current advances show that gastric cancer therapeutic strategies should focus on early diagnosis and operation therapy, enhanced immunotherapy, imagingdirected therapy, and killing gastric cancer stem cells to overcome multidrug resistance (MDR). Given these strategies, we designed and prepared some nanodevices and series of multifunctional nanoprobes for screening, prewarning, early diagnosis and targeted imaging and therapy of early gastric cancer, including prewarning SNP arrays, breath analysis sensors, CTC microfluidic chips, series of fluorescent magnetic nanoprobes, series of quantum dots nanoprobes [52], series of gold nanoprobes, series of upconversion nanoprobes, RNA nanoprobes, and nanoprobes for killing gastric cancer stem cells and enhanced immunotherapeutic efficacy. The main challenges to the translational research of those nanoprobes still focus on the better understanding of the interactions between nanoparticles and biomacromolecules and the immune system, the homogeneity of the material preparations, the paucity of the knowledge regarding pertinent biomarkers and the concern regarding biocompatibility. Green synthesis of nanoparticles, multimodality molecular imaging, SERS analysis and theranostics are particular fields of interest. However, designers must possess a systematic view from the beginning as they approach the goal of nanoprobes for translational medical research [53-56]. We believe that nano-theransotic devices and multifunctional nanoprobes will serve the patients in near future.

\section{Acknowledgements}

This work was supported by Chinese Key Basic Research Pro-gram (973 Project) (No. 2010 CB933901 and No. 2015CB931802), the National Natural Scientific Foundation of China (Grant No. 81225010, 81327002, and 31170961), and 863 project of China (no. 2014AA020700), Shanghai Science and Technology Fund (No. 13NM1401500).

\section{References}

[1] J. Ferlay, H.R. Shin, F. Bray, et al., Estimates of worldwide burden of cancer in 2008: GLOBOCAN 2008. Int. J. Cancer, 2010, 127: 2893-2917.

[2] A. Jemal, F. Bray, M.M. Center, et al., Global cancer statistics. CA Cancer J. Clin., 2011, 61: 69-90.

[3] T. Takahashi, Y. Saikawa and Y. Kitagawa, Gastric cancer: current status of diagnosis and treatment. Cancers (Basel), 2013, 5: 48-63.

[4] B.J. Dicken, D.L. Bigam, C. Cass, et al., Gastric adenocarcinoma: review and considerations for future directions. Annals of Surgery, 2005, 241: 27-39.

[5] N. Uemura, S. Okamoto, S. Yamamoto, et al., Helicobacter pylori infection and the development of gastric cancer. New England Journal of Medicine, 2001, 345: 784-789

[6] R.L. Comis, S.K. Carter, A review of chemotherapy in gastric cancer. Cancer, 1974, 34: 1576-1586

[7] B.Z. Shen, Systems molecular imaging: right around the corner. Nano Biomed. Eng., 2014, 6: 1-5.

[8] S. Cheng, D. Cui, Accurate non-rigid registration of lung images based on mutual information. Nano Biomed. Eng., 2015, 7(4): 153-159.

[9] C.Y. Kuo, Y. Chao and C.P. Li, Update on treatment of gastric cancer. Journal of the Chinese Medical Association, 2014, 77(7): 345-353.

[10] I. Proserpio, S. Rausei, S. Barzaghi, et al., Multimodal treatment of gastric cancer. World Journal of Gastrointestinal Surgery, 2014, 6: 55-58

[11] D. Zhang, D. Fan, New insights into the mechanisms of gastric cancer multidrug resistance and future perspectives. Future Oncol., 2010, 6: 527-537.

[12] D. Cui, L. Zhang, X. Yan, et al., A microarraybased gastric carcinoma prewarning system. World $J$ Gastroenterol, 2005, 11: 1273-1282.

[13] Y. Zhang, G. Gao, H. Liu, et al., Identification of volatile biomarkers of gastric cancer cells and ultrasensitive electrochemical detection based on sensing interface of Au-Ag alloy coated MWCNTs. Theranostics, 2014, 4: 154.

[14] K. Wang, J. Ruan, Q. Qian, et al., BRCAA1 monoclonal antibody conjugated fluorescent magnetic nanoparticles for in vivo targeted magnetofluorescent imaging of gastric cancer. J Nanobiotechnol, 2011, 9: 23.

[15] J. Ruan, H. Song, Q. Qian, et al., HER2 monoclonal antibody conjugated RNase-A-associated CdTe quantum dots for targeted imaging and therapy of gastric cancer. Biomaterials, 2012, 33: 7093-7102. 
[16] X. Mou, T. Li, J. Wang, et al., Genetic variation of BCL2 (rs2279115), NEIL2 (rs804270), LTA (rs909253), PSCA (rs2294008) and PLCE1 (rs3765524, rs10509670) genes and their correlation to gastric cancer risk based on universal tagged arrays and $\mathrm{Fe} 3 \mathrm{O} 4$ magnetic nanoparticles. Journal of Biomedical Nanotechnology, 2015, 11: 2057-2066.

[17] Y. Chen, Y. Zhang, F. Pan, et al., Breath analysis based on surface enhanced Raman scattering sensor distinguishes early and advanced gastric cancer patients from healthy persons. ACS Nano, 2016, 10(9): 8169-8179.

[18] Y. Xie, X. Zhi, H. Su, et al., A novel electrochemical microfluidic chip combined with multiple biomarkers for early diagnosis of gastric cancer. Nanoscale Res. Lett., 2015, 10: 477.

[19] L. Chen, C. Bao, C. Lei, et al., A prototype of giant magnetoimpedance-based biosensing system for targeted detection of gastric cancer cells. Biosensors \& Bioelectronics, 2011, 26: 3246-3253.

[20] C. Bao, L. Chen, T. Wang, et al., One step quick detection of cancer cell surface marker by integrated NiFe-based magnetic biosensing cell cultural chip. Nano-Micro Letters, 2013, 5: 213-222

[21] Y. Liu, M. Yang, J. Zhang, et al., Human induced pluripotent stem cells for tumor targeted delivery of gold nanorods and enhanced photothermal therapy. ACS Nano, 2016, 10: 2375-2385.

[22] C. Bao, J. Conde, F. Pan, et al., Gold nanoprisms as a hybrid in vivo cancer theranostic platform for in situ photoacoustic imaging, angiography, and localized hyperthermia. Nano Research, 2016, 9: 1043-1056.

[23] C. Bao, J. Conde, J. Curtin, et al., Bioresponsive antisense DNA gold nanobeacons as a hybrid in vivo theranostics platform for the inhibition of cancer cells and metastasis. Sci. Rep., 2015, 12297.

[24] D. Shu, W.D. Moll, Z. Deng, et al., Bottom-up assembly of RNA arrays and superstructures as potential parts in nanotechnology. Nano Letters, 2004, 4: 1717-1723

[25] L. Qi, L. Wu, S. Zheng, et al., Cell-penetrating magnetic nanoparticles for highly efficient delivery and intracellular imaging of siRNA. Biomacromolecules, 2012, 13: 27232730.

[26] E.A. Murphy, B.K. Majeti, R. Mukthavaram, et al., Targeted nanogels: a versatile platform for drug delivery to tumors. Molecular Cancer Therapeutics, 2011, 10: 972-982.

[27] X. Yu, M.V. Pishko, Nanoparticle-based biocompatible and targeted drug delivery: characterization and in vitro studies. Biomacromolecules, 2011, 12: 3205-3212.

[28] J. Ma, Z. Zhou, C. Zhang, et al., Folic acid-conjugated $\mathrm{LaF}_{3}: \mathrm{Yb}, \mathrm{Tm} @ \mathrm{SiO}_{2}$ nanoprobes for targeting dualmodality imaging of upconversion luminescence and X-ray computed tomography. Journal Physical Chemistry C, 2012, 116: 14062-14070.

[29] K.R. Kalli, A.L. Oberg, G.L. Keeney, et al., Folate receptor alpha as a tumor target in epithelial ovarian cancer. Gynecologic Oncology, 2008, 108: 619-626.

[30] S. Liang, C. Li, C. Zhao, et al., CD44v6 monoclonal antibody-conjugated gold nanostars for targeted photoacoustic imaging and plasmonic photothermal therapy of gastric cancer stem-like cells. Theranostics, 2015, 5: 879-881.

[31] S. Vinogradov, X. Wei, Cancer stem cells and drug resistance: The potential of nanomedicine. Nanomedicine (Lond), 2012, 7: 597-615.

[32] D. Zhang, D. Fan, New insights into the mechanisms of gastric cancer multidrug resistance and future perspectives. Future Oncol., 2010, 6: 527-537.

[33] M.F. Clarke, J.E. Dick, P.B. Dirks, et al., Cancer stem cells-perspectives on current status and future directions:
Aacr workshop on cancer stem cells. Cancer Res., 2006, 66: 9339-9344.

[34] R.J. Gilbertson, T.A. Graham, Cancer: resolving the stemcell debate. Nature, 2012, 488: 462-463.

[35] S. Takaishi, T. Okumura, S. Tu, et al., Identification of gastric cancer stem cells using the cell surface marker cd44. Stem Cells, 2009, 27: 1006-1020.

[36] D. Naor, R.V. Sionov, D. Ish-Shalom, Cd44: structure, function, and association with the malignant process. Adv Cancer Res., 1997, 71: 241-319.

[37] C. Zhang, C. Li, Y. Liu, et al., Folic acid/ce6 conjugated gold nanoclusters for NIR fluorescent imaging and photodynamic therapy with enhanced permission and retention. Adv. Fun. Mater., 2015, 28: 1314-1325.

[38] S. Cheng, D. Cui, Accurate non-rigid registration of lung images based on mutual information. Nano Biomed. Eng., 2015, 7(4): 153-159.

[39] F. Chen, P. Huang, Y. Zhu, et al., The photoluminescence, drug delivery and imaging properties of multifunctional $\mathrm{Eu}^{3+} / \mathrm{Gd}^{3+}$ dual-doped hydroxyapatite nanorods. Biomaterials, 2011, 32: 9031-9039.

[40] D. Zhang, D. Fan, New insights into the mechanisms of gastric cancer multidrug resistance and future perspectives. Future Oncol., 2010, 6: 527-537.

[41] M.F. Clarke, J.E. Dick, P.B. Dirks, et al., Cancer stem cells-perspectives on current status and future directions: AACR workshop on cancer stem cells. Cancer Res., 2006; 66: 9339-9344.

[42] E.F. Khisamutdinov, D.L. Jasinski and P. Guo, RNA as a boiling-resistant anionic polymer material to build robust structures with defined shape and stoichiometry. ACS Nano, 2014, 8: 4771-4781.

[43] D. Cui, C. Zhang, B. Liu, et al., Regression of gastric cancer by Systemic Injection of RNA Nanoparticles Carrying both Ligand and siRNA. Scientific Report, 2015, 5: 10732 .

[44] X. Wang, L. Yang, Z. Chen, Application of nanotechnology in cancer therapy and imaging. $C A$ Cancer J Clin., 2008, 58: 97-110.

[45] Y. Yang, J. Zhang, F. Xia, et al., Human CIK cells loaded with Au nanorods as a theranostic platform for targeted photoacoustic imaging and enhanced immunotherapy and photothermal therapy. Nanoscale Res. Lett., 2016, 11: 285.

[46] L. Lu, G. Yan, K. Zhao, et al., An implantable telemetry platform system with ASIC for in vivo monitoring of gastrointestinal physiological information. IEEE Sensors Journal, 2015, 12: 3524-3534.

[47] R. Weissleder, A clearer vision for in vivo imaging. Nat Biotechnol, 2001, 19: 316-317.

[48] B. Pan, D. Cui, P. Xu, et al., Synthesis and characterization of polyamidoamine dendrimer-coated multi-walled carbon nanotubes and their application in gene delivery systems. Nanotechnology, 2009, 20: 125101.

[49] P. Huang, C. Xu, J. Lin, et al., Folic acid-conjugated graphene oxide loaded with photosensitizers for targeting photodynamic therapy. Theranostics, 2011, 1: 240-250.

[50] Z. Li, P. Huang, X. Zhang, et al., RGD-conjugated dendrimer-modified gold nanorods for in vivo tumor targeting and photothermal therapy. Mol Pharm., 2010, 7: 94-104.

[51] L. Nie, X. Chen, Structural and functional photoacoustic molecular tomography aided by emerging contrast agents. Chem Soc Rev., 2014, 43: 7132-7170.

[52] I. Venugopal, S. Pernal, T. Fusinatto, et al., Quantum dot conjugated magnetic nanoparticles for targeted drug delivery and imaging. Nano Biomed. Eng., 2016, 8: 2438.

[53] W. Li, X. Sun, Y. Wang, et al., In vivo quantitative photoacoustic microscopy of gold nanostar kinetics in 
mouse organs. Biomedical Optics Express, 2014, 5: 26792685.

[54] L. Nie, P. Huang, W. Li, et al., Early-stage imaging of nanocarrier-enhanced chemotherapy response in living subjects by scalable photoacoustic microscopy. ACS Nano, 2014, 8: 12141-12150.

[55] E.A. Sykes, J. Chen, G. Zheng, et al., Investigating the impact of nanoparticle size on active and passive tumor targeting efficiency. ACS Nano, 2014, 8: 5696-5706.

Copyright $₫ 2016$ Daxiang Cui, Lijun Ma, Xiao Zhi and Chunlei Zhang. This is an open-access article distributed under the terms of the Creative Commons Attribution License, which permits unrestricted use, distribution, and reproduction in any medium, provided the original author and source are credited. 